Article

\title{
Integrative Genomic and Transcriptomic Profiling Reveals a Differential Molecular Signature in Uterine Leiomyoma versus Leiomyosarcoma
}

\author{
Alba Machado-Lopez ${ }^{1}\left(\mathbb{D}\right.$, Roberto Alonso ${ }^{1,2} \mathbb{D}$, Victor Lago ${ }^{3}$, Jorge Jimenez-Almazan ${ }^{2} \mathbb{D}$, Marta Garcia $^{2}$, \\ Javier Monleon ${ }^{4}$, Susana Lopez ${ }^{5}$, Francisco Barcelo ${ }^{6}$, Amparo Torroba ${ }^{7}$, Sebastian Ortiz ${ }^{8}$, Santiago Domingo ${ }^{3}$, \\ Carlos Simon $1,9,10, *,+$ and Aymara Mas $1, *,+(\mathbb{D}$
}

check for updates

Citation: Machado-Lopez, A.; Alonso, R.; Lago, V.; Jimenez-Almazan, J.; Garcia, M.; Monleon, J.; Lopez, S.; Barcelo, F.; Torroba, A.; Ortiz, S.; et al. Integrative Genomic and Transcriptomic Profiling Reveals a Differential Molecular Signature in Uterine Leiomyoma versus Leiomyosarcoma. Int. J. Mol. Sci. 2022, 23, 2190. https://doi.org/ $10.3390 /$ ijms 23042190

Academic Editor: Andrea Tinelli

Received: 18 January 2022

Accepted: 11 February 2022

Published: 16 February 2022

Publisher's Note: MDPI stays neutral with regard to jurisdictional claims in published maps and institutional affiliations.

Copyright: (C) 2022 by the authors. Licensee MDPI, Basel, Switzerland. This article is an open access article distributed under the terms and conditions of the Creative Commons Attribution (CC BY) license (https:// creativecommons.org/licenses/by/ $4.0 /)$.
1 Igenomix Foundation, INCLIVA Biomedical Research Institute, 46980 Valencia, Spain; alba.machado@igenomix.com (A.M.-L.); roberto.alonso@igenomix.com (R.A.)

2 Research and Development Department, Igenomix SL, 46980 Paterna, Spain; jorge.jimenez@igenomix.com (J.J.-A.); marta.garcia@igenomix.com (M.G.)

3 Gynecologic Oncology Department, University Hospital La Fe, 46026 Valencia, Spain; victor.lago.leal@hotmail.com (V.L.); santiago.domingo.delpozo@gmail.com (S.D.)

4 Department of Obstetrics and Gynecology, Hospital Universitario La Fe, 46026 Valencia, Spain; monlesancho@gmail.com

5 Department of Pathology, Hospital Universitario La Fe, 46026 Valencia, Spain; susana.lopezagullo@hotmail.com

6 Department of Gynecology and Obstetrics, Gynecology Oncology Unit, Hospital Universitario Virgen de la Arrixaca, 30120 Murcia, Spain; fjbarcelovalcarcel@hotmail.com

7 Pathology Service, Hospital Clínico Universitario Virgen de la Arrixaca, 30120 Murcia, Spain; mariaa.torroba@carm.es

8 Department of Pathology, Complejo Hospitalario de Cartagena, 30202 Murcia, Spain; sortizreina@yahoo.es

9 Department of Obstetrics and Gynecology, Universidad de Valencia, 46010 Valencia, Spain

10 Department of Obstetrics and Gynecology, BIDMC, Harvard University, Boston, MA 02215, USA

* Correspondence: carlos.simon@igenomix.com (C.S.); aymara.mas@igenomix.com (A.M.); Tel.: +34-963-90-53-10 (C.S. \& A.M.)

+ These authors should be considered joint senior authors.

\begin{abstract}
The absence of standardized molecular profiling to differentiate uterine leiomyosarcomas versus leiomyomas represents a current diagnostic challenge. In this study, we aimed to search for a differential molecular signature for these myometrial tumors based on artificial intelligence. For this purpose, differential exome and transcriptome-wide research was performed on histologically confirmed leiomyomas $(n=52)$ and leiomyosarcomas $(n=44)$ to elucidate differences between and within these two entities. We identified a significantly higher tumor mutation burden in leiomyosarcomas vs. leiomyomas in terms of somatic single-nucleotide variants $(171,863$ vs. 81,152$)$, indels (9491 vs. 4098), and copy number variants (8390 vs. 5376). Further, we discovered alterations in specific copy number variant regions that affect the expression of some tumor suppressor genes. A transcriptomic analysis revealed 489 differentially expressed genes between these two conditions, as well as structural rearrangements targeting ATRX and RAD51B. These results allowed us to develop a machine learning approach based on 19 differentially expressed genes that differentiate both tumor types with high sensitivity and specificity. Our findings provide a novel molecular signature for the diagnosis of leiomyoma and leiomyosarcoma, which could be helpful to complement the current morphological and immunohistochemical diagnosis and may lay the foundation for the future evaluation of malignancy risk.
\end{abstract}

Keywords: leiomyoma; leiomyosarcoma; exome/transcriptome; mutational pattern; differential gene expression; integrative analysis; diagnostic/prognostic biomarkers; machine learning; classification model 


\section{Introduction}

Uterine leiomyomas (LM) are benign tumors arising in the smooth muscle cells of the uterine wall. They are the most common pelvic tumors in women, with a prevalence of $>80 \%$ for African American and $\sim 70 \%$ for Caucasian women before 50 years of age [1] Although LM are non-malignant tumors, the risk of hidden undiagnosed malignancy, such as leiomyosarcoma (LMS), occurs in one among 498 uterine tumors [2-4].

Histological diagnosis is the gold standard option for myometrial tumors [5,6]. However, LM and LMS share clinical symptoms and morphological features $[7,8]$, sometimes hindering their differential diagnosis and introducing the risk of the future potential spread of undiagnosed LMS with the use of power morcellators [9]. Besides, alternative invasive approaches, such as laparotomy-based procedures, increase morbidity, mortality, and cost for the patient and healthcare system [10].

Despite LM and LMS having been previously characterized at a molecular level [11-13], the differential profiling of these myometrial tumors based on genomic/transcriptomic characteristics could allow us a better understanding of the underlying tumorigenic processes as well as to develop novel tools that may aid the current clinical diagnosis. Given these challenges, we aim to discover specific molecular signatures for the differential diagnosis of myometrial tumors.

In this study, we identified that LM and LMS have significant mutational heterogeneity and differences in copy number alterations at the DNA level, while a specific transcriptomic profile and multiple structural rearrangements were detected at the RNA level. With these data, an integrated molecular analysis was performed to assess the effect of copy number variants (CNVs) on gene expression. Targeted RNAseq data and artificial intelligence were used to create a predictive model for the comprehensive molecular classification of LMS and LM at the tumor-tissue level.

\section{Results}

\subsection{Clinical Study Design}

After obtaining informed consent from the eligible patients, tumor samples were collected from women undergoing a hysterectomy or myomectomy as a surgical treatment for a primary myometrial tumor. Following histological diagnoses according to WHO criteria [14], a total of 106 selected LM and LMS samples were separated in two cohorts, the experimental cohort and the validation cohort (Supplementary Figure S1). The epidemiological, histopathological, and clinical outcomes of the patients involved in the study are summarized in Table 1.

Table 1. Epidemiological, demographic, and clinicopathological outcomes of 56 patients diagnosed with uterine leiomyoma (LM) and 47 patients with leiomyosarcoma (LMS) from the experimental cohort.

\begin{tabular}{|c|c|c|c|}
\hline & Characteristics & LMS & LM \\
\hline \multirow{16}{*}{ 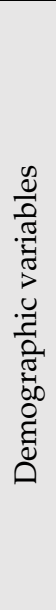 } & Age & & \\
\hline & $\leq 30$ years & - & $2(3.57 \%)$ \\
\hline & $31-40$ years & $7(14.89 \%)$ & $17(30.36 \%)$ \\
\hline & $41-50$ years & $11(23.41 \%)$ & $33(58.93 \%)$ \\
\hline & $51-60$ years & $20(42.55 \%)$ & $2(3.57 \%)$ \\
\hline & $\geq 61$ years & $9(19.15 \%)$ & - \\
\hline & Not available $(n)$ & - & $2(3.57 \%)$ \\
\hline & Median (years) & 53 & 44 \\
\hline & Range (years & $35-75$ & $28-55$ \\
\hline & Ethnicity & & \\
\hline & Caucasian & $36(76.59 \%)$ & $41(73.21 \%)$ \\
\hline & African American & $1(2.13 \%)$ & $1(1.79 \%)$ \\
\hline & Latin & $4(8.51 \%)$ & $6(10.71 \%)$ \\
\hline & Asian & $1(2.13 \%)$ & - \\
\hline & Arabic & $1(2.13 \%)$ & - \\
\hline & Not available & $4(8.51 \%)$ & $8(14.29 \%)$ \\
\hline
\end{tabular}


Table 1. Cont.

\begin{tabular}{|c|c|c|c|}
\hline & Characteristics & LMS & $\overline{\text { LM }}$ \\
\hline & Body mass index $\left(\mathrm{kg} / \mathrm{m}^{2}\right)$ & & \\
\hline & Median & 27.15 & 24 \\
\hline & Range & $21.5-34.9$ & $18.20-34.63$ \\
\hline & Not available $(n)$ & 21 & 13 \\
\hline \multirow{12}{*}{ 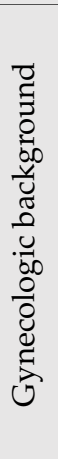 } & Parity & & \\
\hline & Yes & $23(48.94 \%)$ & $27(48.21 \%)$ \\
\hline & No & - & $1(1.79 \%)$ \\
\hline & Not available & $24(51.06 \%)$ & $28(50.00 \%)$ \\
\hline & Miscarriage & & \\
\hline & Yes & $7(14.89 \%)$ & $15(26.79 \%)$ \\
\hline & No & $16(34.05 \%)$ & $13(23.21 \%)$ \\
\hline & Not available & $24(51.06 \%)$ & $28(50.00 \%)$ \\
\hline & Menopausal status & & \\
\hline & Premenopausal & $15(38.30 \%)$ & $46(82.14 \%)$ \\
\hline & Postmenopausal & $18(31.91 \%)$ & $2(3.57 \%)$ \\
\hline & Not available & $14(29.79 \%)$ & $8(14.29 \%)$ \\
\hline \multirow{12}{*}{$\begin{array}{l}\text { है } \\
\text { ई } \\
\text { है } \\
\text { ڤે }\end{array}$} & \multicolumn{3}{|l|}{ Pelvic mass } \\
\hline & Yes & $25(53.19 \%)$ & $28(50.00 \%)$ \\
\hline & No & $7(14.89 \%)$ & $20(35.71 \%)$ \\
\hline & Not available & $15(31.92 \%)$ & $8(14.29 \%)$ \\
\hline & Abnormal uterine bleeding & & \\
\hline & Yes & $17(36.17 \%)$ & $26(46.43 \%)$ \\
\hline & No & $11(23.40 \%)$ & $21(37.50 \%)$ \\
\hline & Not available & $19(40.43 \%)$ & $9(16.07 \%)$ \\
\hline & Abdominal pain & & \\
\hline & Yes & $16(34.04 \%)$ & $14(25.00 \%)$ \\
\hline & No & $11(23.41 \%)$ & $32(57.14 \%)$ \\
\hline & Not available & $20(42.55 \%)$ & $10(17.86 \%)$ \\
\hline \multirow{20}{*}{ 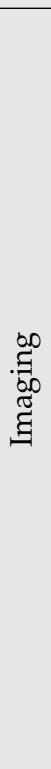 } & \multicolumn{3}{|l|}{$\mathrm{CT}$} \\
\hline & Yes & $14(29.79 \%)$ & $7(12.50 \%)$ \\
\hline & No & $16(34.04 \%)$ & $42(75.00 \%)$ \\
\hline & Not available & $17(36.17 \%)$ & $7(12.50 \%)$ \\
\hline & \multicolumn{3}{|l|}{ MRI } \\
\hline & Yes & $5(10.64 \%)$ & $5(8.93 \%)$ \\
\hline & No & $22(46.81 \%)$ & $44(78.57 \%)$ \\
\hline & Not available & $20(42.55 \%)$ & $7(12.50 \%)$ \\
\hline & \multicolumn{3}{|l|}{ Ultrasound } \\
\hline & Yes & $31(65.96 \%)$ & $49(87.50 \%)$ \\
\hline & No & - & - \\
\hline & Not available & $16(34.04 \%)$ & $7(12.50 \%)$ \\
\hline & \multicolumn{3}{|l|}{ Tumor size $(\mathrm{cm})$} \\
\hline & Median & 13 & 7.4 \\
\hline & Range & mar-24 & $0.25-25$ \\
\hline & Not available $(n)$ & 17 & 10 \\
\hline & Suspected uterine sarcoma & & \\
\hline & Yes & $15(31.91 \%)$ & $5(8.93 \%)$ \\
\hline & No & $15(31.91 \%)$ & $44(78.57 \%)$ \\
\hline & NA & $17(36.18 \%)$ & $7(12.50 \%)$ \\
\hline \multirow{10}{*}{ 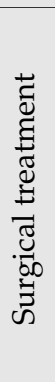 } & \multicolumn{3}{|l|}{ Endometrial biopsy } \\
\hline & Yes & $15(31.91 \%)$ & $39(69.64 \%)$ \\
\hline & No & $17(27.66 \%)$ & $10(17.86 \%)$ \\
\hline & Not available & $19(40.43 \%)$ & $7(12.50 \%)$ \\
\hline & \multicolumn{3}{|l|}{ Primary surgery } \\
\hline & Laparoscopic hysterectomy & $1(2.13 \%)$ & $12(21.43 \%)$ \\
\hline & Laparoscopic myomectomy & - & $5(8.93 \%)$ \\
\hline & Laparotomic hysterectomy & $33(70.21 \%)$ & $21(37.50 \%)$ \\
\hline & Laparotomic myomectomy & - & $11(19.64 \%)$ \\
\hline & Not available & $13(27.66 \%)$ & $7(12.50 \%)$ \\
\hline
\end{tabular}


Table 1. Cont.

\begin{tabular}{|c|c|c|c|}
\hline & Characteristics & LMS & $\overline{\mathbf{L M}}$ \\
\hline \multirow{12}{*}{ 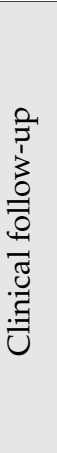 } & Recurrence & & \\
\hline & Yes & $19(40.43 \%)$ & $49(87.50 \%)$ \\
\hline & No & $9(19.16 \%)$ & - \\
\hline & Not available & $19(40.43 \%)$ & $7(12.50 \%)$ \\
\hline & Status & & \\
\hline & Alive & $12(25.53 \%)$ & $48(85.71 \%)$ \\
\hline & Deceased & $12(25.53 \%)$ & - \\
\hline & Not available & $23(48.84 \%)$ & $8(14.29 \%)$ \\
\hline & Follow-up (months) & & \\
\hline & Median & 24 & - \\
\hline & Range & 8-116 & - \\
\hline & Not available $(n)$ & 29 & - \\
\hline
\end{tabular}

\subsection{Identification of Differential Somatic Single Nucleotide Variants and Insertions/Deletions}

We performed whole-exome sequencing in $44 \mathrm{LM}$ and $34 \mathrm{LMS}$ tumors to screen for single nucleotide variants (SNVs) and insertions/deletions (indels). We detected 181,354 small variants in the LMS tumors, of which 171,863 were SNVs and 9491 were small indels. In the LM samples, we detected 85,250 small variants, of which 81,152 were SNVs and 4098 were small indels. Among these variants, $27.63 \%$ where shared between LMS and LM, while $34.56 \%$ were LMS-exclusive and $37.81 \%$ were LM-exclusive (in at least one sample).

Although comparative analyses of SNVs showed a similar distribution, LMS had a higher mean number of alterations per sample compared to LM $(p<0.05)$. Then, we focused on group-exclusive variants present in at least six samples of each group to find exclusive variants involved in the pathogenic process of each group. As a result, we found a total of thirteen variants affecting eight different genes exclusive to LMS (Figure 1A), while we found a total of twelve mutations affecting twelve genes exclusive to LM (Figure 1B). In both groups, these mutations were mostly missense mutations, although, in the LMS group, there were other types of variants, i.e., in frame indels affecting the IQCJ-SCHIPI gene, structural interaction variants affecting the GAPDH gene, and frameshift variants affecting the EEF2 gene. Interestingly, most of the variants detected have been previously reported in other cancer types in the COSMIC database (Supplementary Table S1).

Using this SNV information, we compared the mutational spectrum for the LM and LMS tumors through the relative contribution of six base substitution types (Figure 1C), which were then decomposed into two distinct mutational signatures (Figure 1D).

In an attempt to relate our findings to known mutational signatures, we searched in the COSMIC database [15] and identified four $(1,5,12$, and 20) out of 30 existing signatures (Figure 1E). Among them, signature 1 results from an endogenous mutational process initiated by spontaneous deamination of 5-methylcytosine, while signature 5 exhibits transcriptional strand-bias for $\mathrm{T}>\mathrm{C}$ substitutions at $\mathrm{ApTpN}$ context. We also identified signature 20, which is associated with defective DNA mismatch repair due to high numbers of small indels at mono/polynucleotide repeats. While these signatures have already been identified across 40 different human cancer types, signature 12 represents a novel mutational signature only present in these uterine tumors, showing similarities to liver cancer and exhibiting a strong transcriptional strand-bias for $\mathrm{T}>\mathrm{C}$ substitutions as additional mutational features (Figure 1E).

Since we detected a molecular phenotype with a defect in the DNA mismatch repair system, we next evaluated microsatellite instability (MSI) status to predict the outcome in LM and LMS tumors, although no differences were found in the number of alleles or the fragment size (Supplementary Figure S2). 


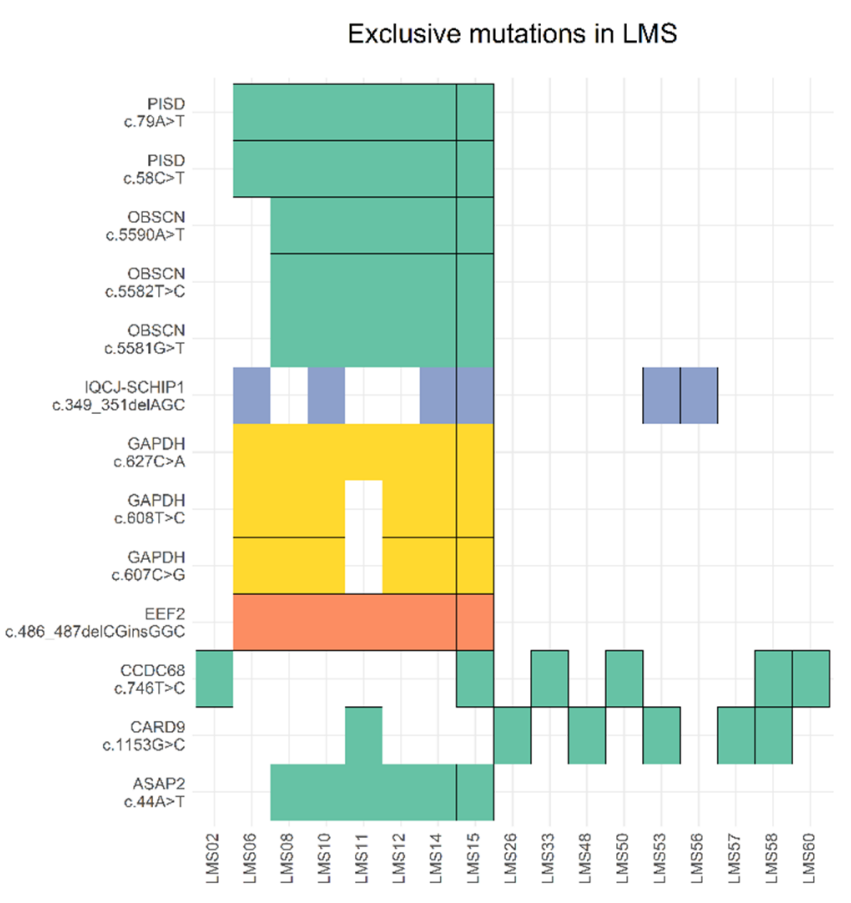

(A)
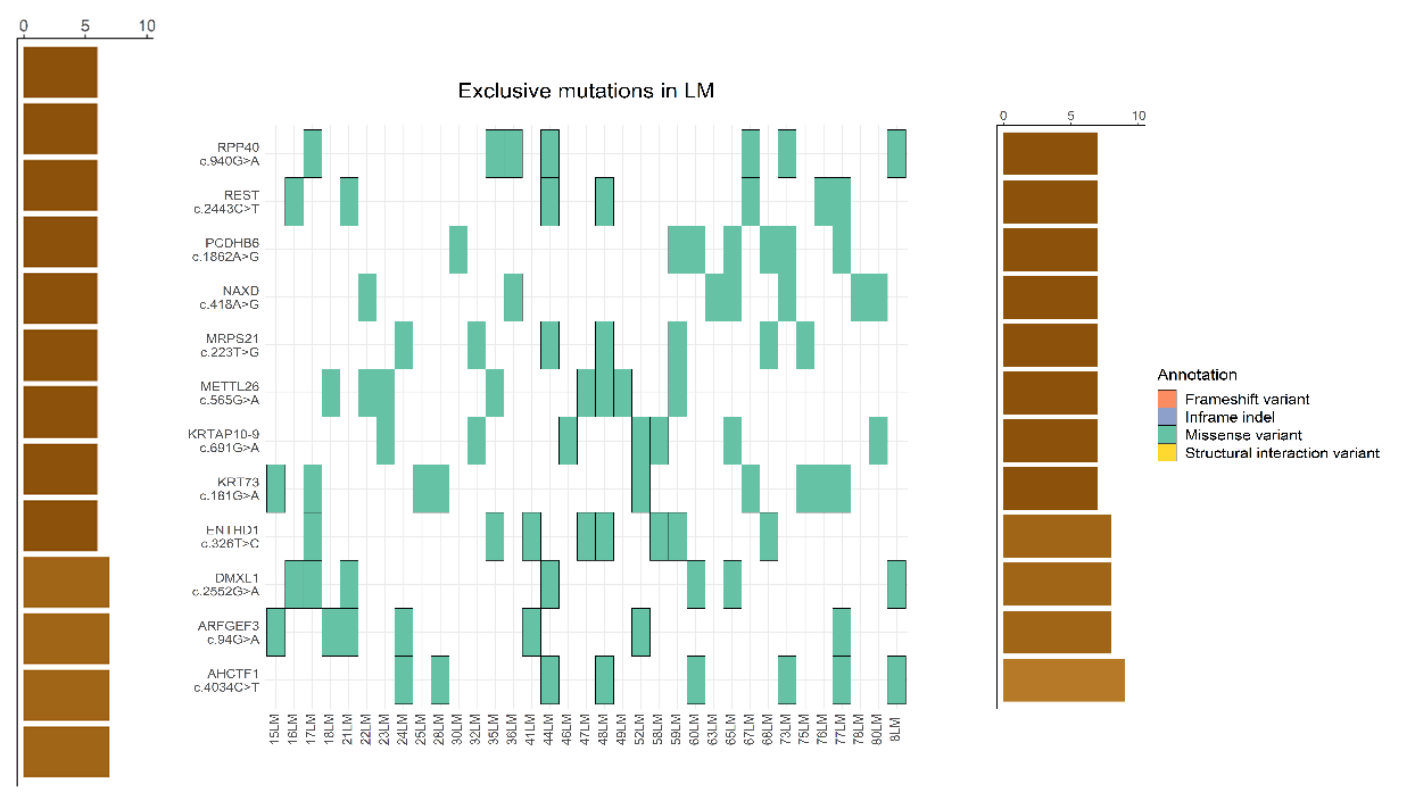

(B)

Figure 1. Cont. 


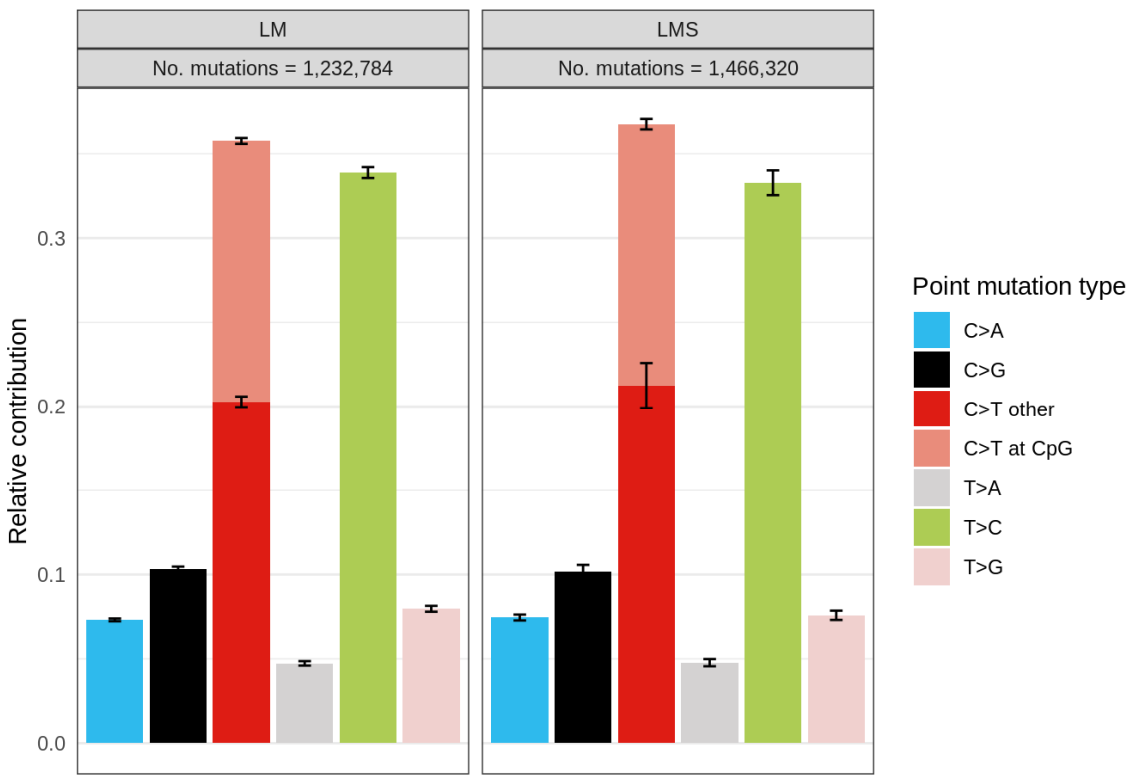

(C)

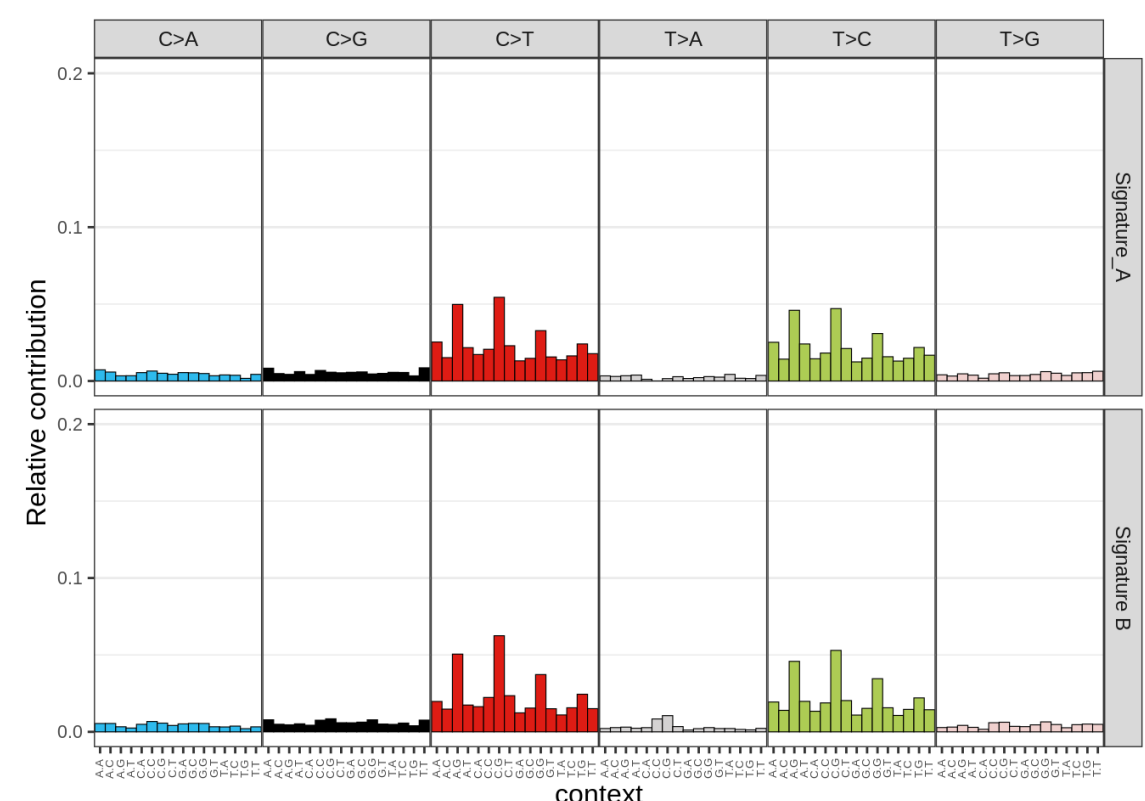

(D)

Figure 1. Cont. 


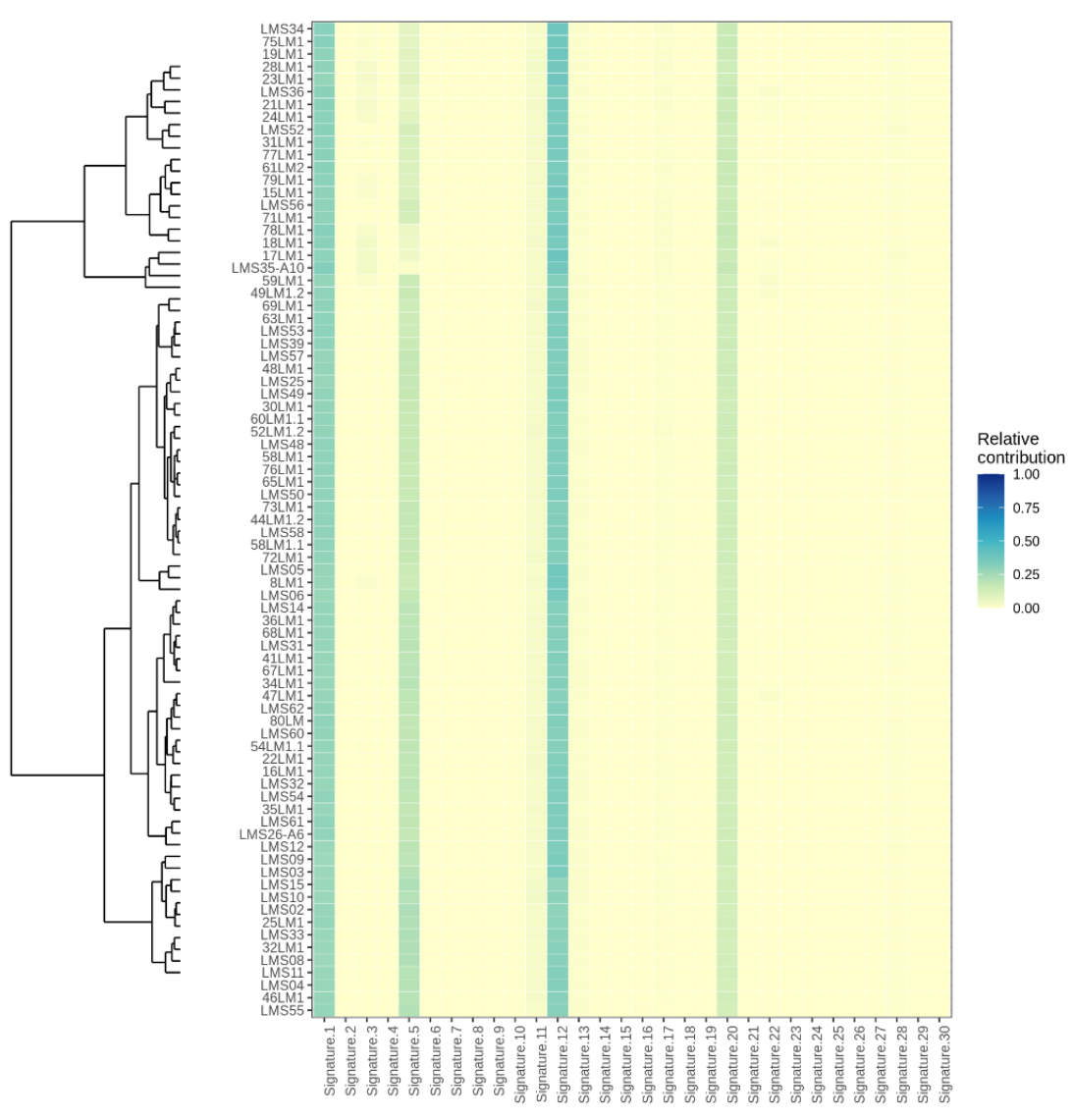

$(\mathrm{E})$

Figure 1. Comparative analysis of single-nucleotide variants (SNVs), insertions/deletions (indels), and mutational signatures for leiomyosarcoma (LMS) and leiomyoma (LM) samples. (A) Tumor profile of LMS-exclusive variants, including frequency and type of mutations. (B) Tumor profile of LM-exclusive variants, including frequency and type of mutations. In both cases, rows represent individual genes, while columns represent individual tumors. Bars illustrate the number of samples for each exclusive mutation. Types of mutations are annotated according to color. (C) Relative contribution of the indicated mutation types to the point mutation spectrum for each tumor type. Error bars indicate standard deviation over all samples. Total number of mutations for LM and LMS is indicated.

(D) Relative contribution of each indicated trinucleotide changes to the two mutational signatures identified by non-negative matrix factorization (NMF) analysis. (E) Heatmap showing relative contribution of each mutational signature described in the COSMIC database for each sample. 


\subsection{Identification of Copy Number Variants}

We next compared the somatic copy number variants (CNVs) in LMS and LM. A total of 14,467 CNVs were detected in LM, while 14,950 CNVs were detected in LMS. Despite the similar results, Student's $t$-test showed a significant difference in the mean values of CNVs per sample between LMS (439.7) and LM (328.8) $\left(p=5.61 \times 10^{-5}\right)$. Because some CNVs were present in more than one sample within each group, we filtered the unique CNVs per group, obtaining a total of 8390 CNVs in LMS and 5376 CNVs in LM. In terms of their structural nature, $18.2 \%$ of the CNVs in LMS were deletions, while $73.1 \%$ were duplications. Specifically, 3.5\% were LMS-specific deletions present in more than one sample, and $5.2 \%$ were LMS-specific duplications present in more than one sample. In the LM group, $11.7 \%$ of the CNVs were deletions and $84.5 \%$ were duplications, with only $0.1 \%$ tumor-specific deletions and $3.6 \%$ tumor-specific duplications (Figure 2A). While the CNV profile for LMS was heterogeneous and showed alterations in most chromosomes, the LM tumors had recurrent losses in chromosomes 1, 13, 14, 15, and 22 and recurrent gains in chromosomes 12 and 19 (Figure 2B).

Kaplan-Meier survival curves were generated to assess the association between LMSspecific CNVs and clinical prognosis based on overall survival. We selected 12 of the most frequent LMS-specific CNVs present in at least 10 out of 34 LMS (Supplementary Table S2) and found statistically significant differences between the patients with disruptions in at least $67 \%$ of the CNVs. Remarkably, we observed a tendency where these patients with aberrant $\mathrm{CNVs}$ had shorter survival times than those with normal copy number values (diploid) in these regions (Figure 2C). To account for possible confounding factors, we performed a multivariate survival analysis, although, as expected, the results were non-significant, probably due to the low sample size and the high number of variables included. Still, given the potential clinical relevance of this finding, unsupervised hierarchical clustering using the 370 genes included in these CNVs demonstrated a clear separation between the LMS and LM tumors at the DNA level (Figure 2D). 


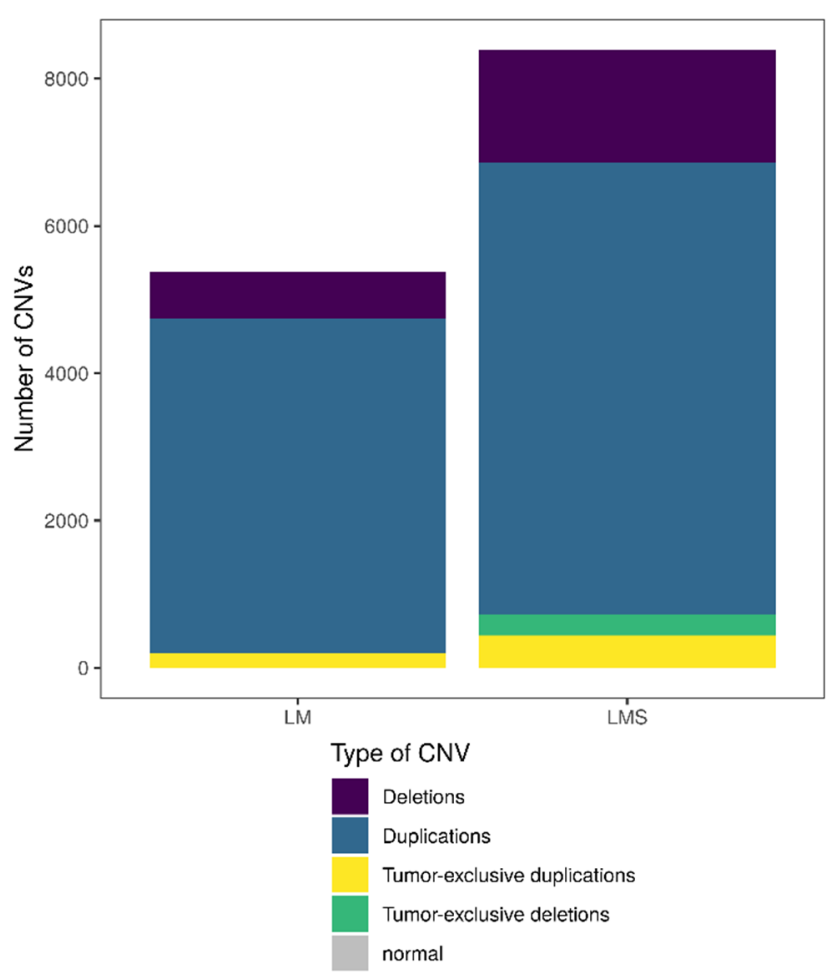

(A)

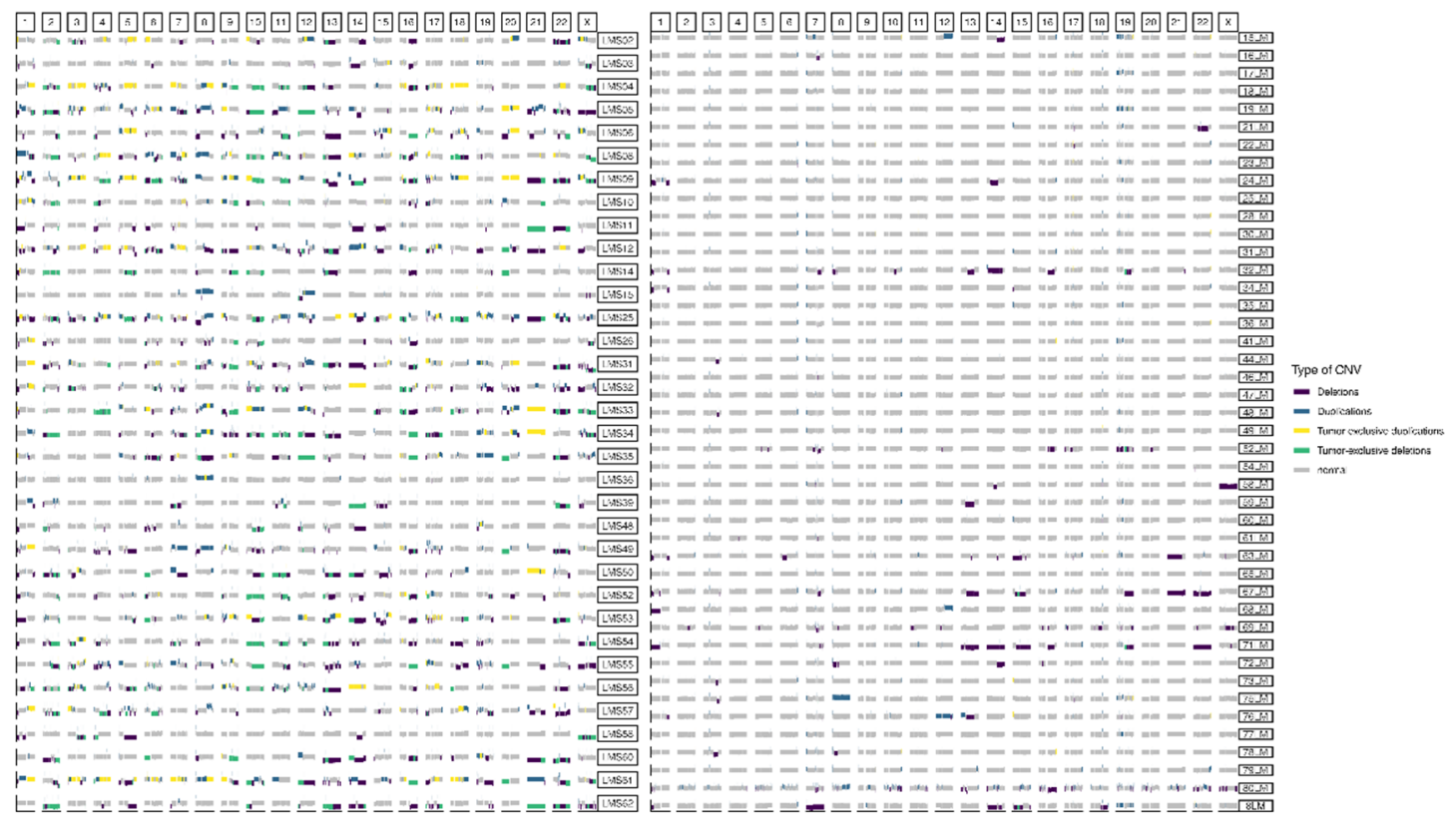

(B)

Figure 2. Cont. 


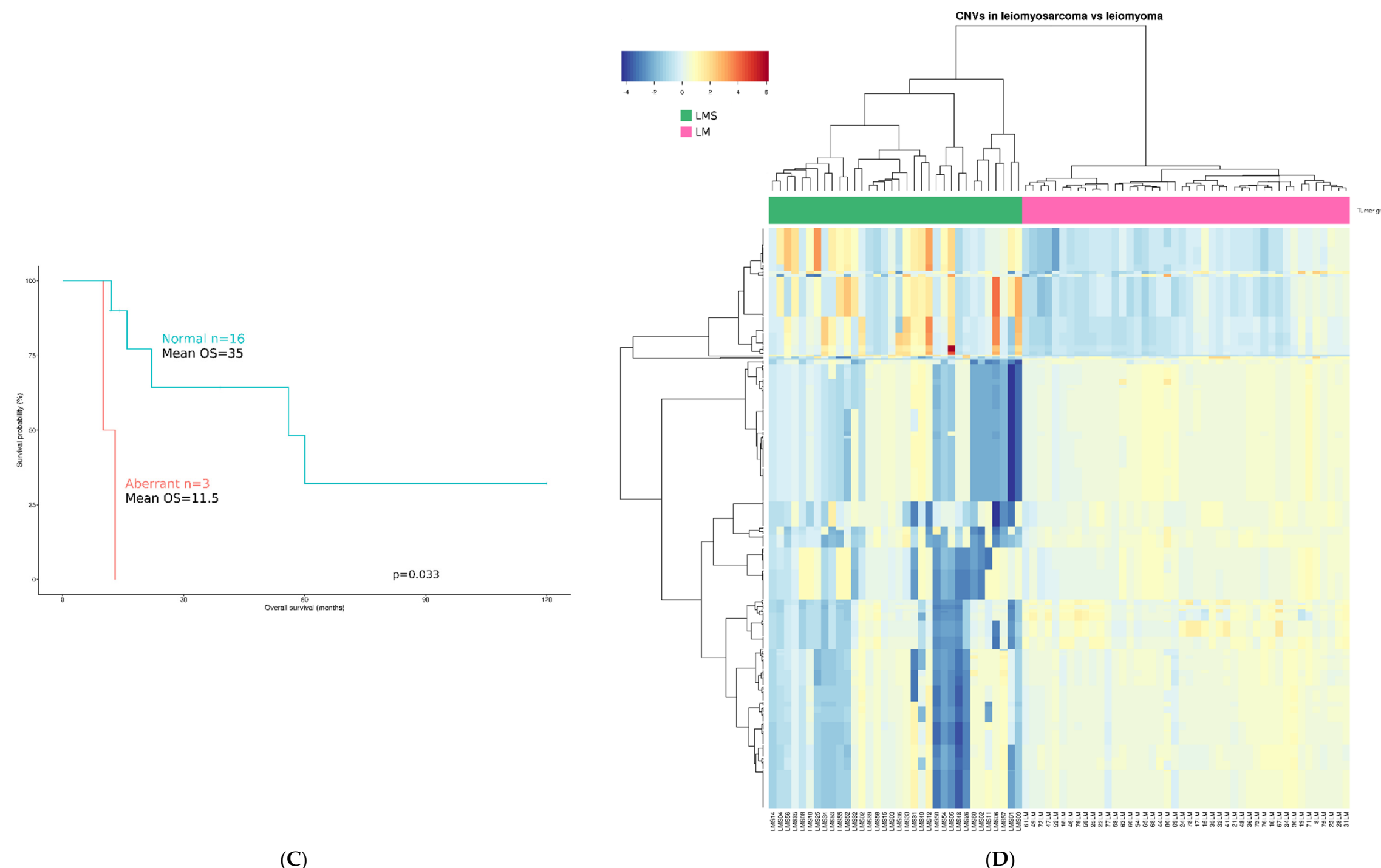

Figure 2. Cont 

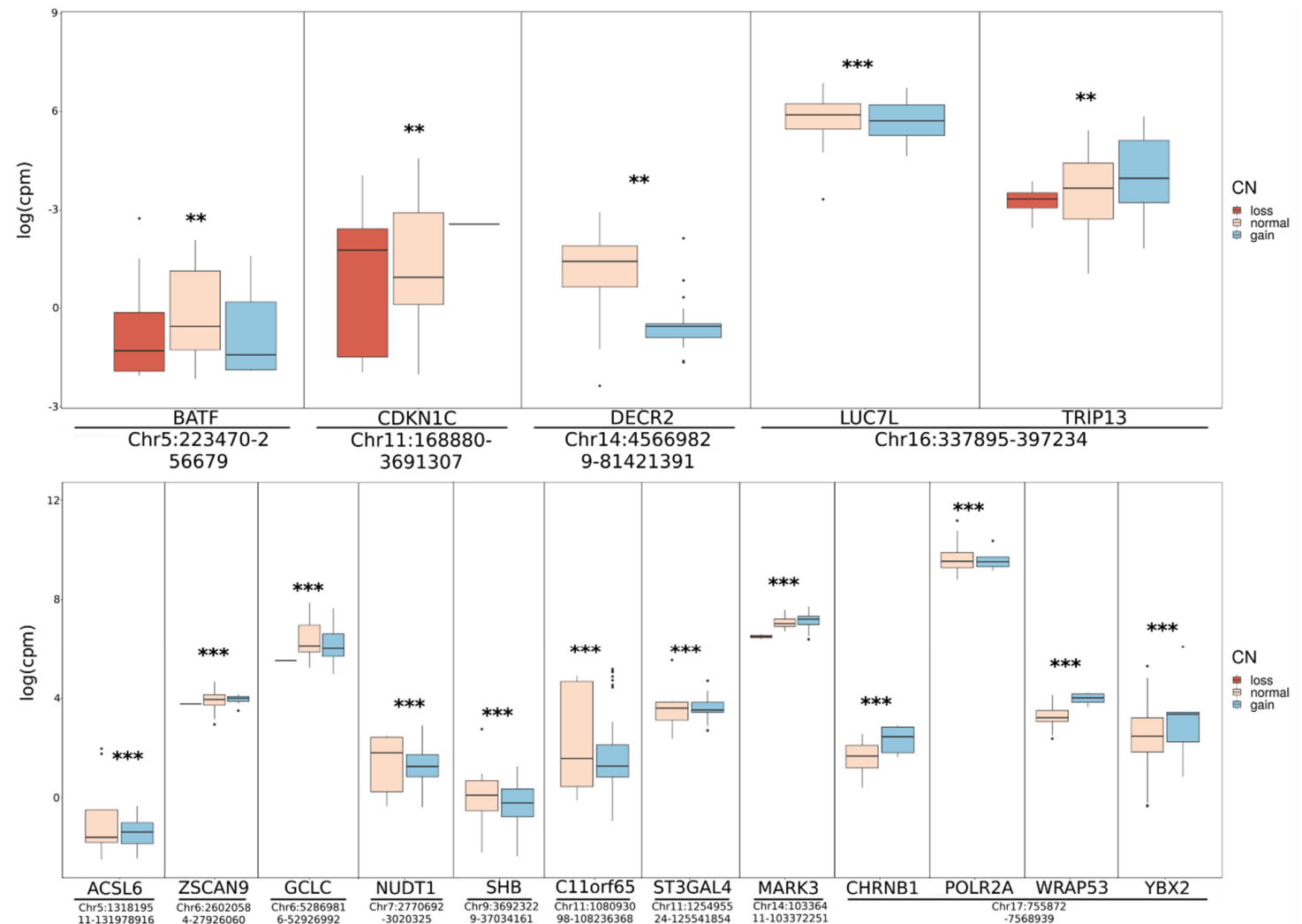

(E)

Figure 2. Comparative analysis of copy number variants (CNVs) in leiomyosarcoma (LMS) and leiomyoma (LM) samples and proximal effects from integrative analysis of CNVs and RNAseq data. (A) Distribution of CNVs per tumor type. (B) Genome-wide CNV distribution in LMS (left) and LM (right). In both cases, rows represent individual samples, while columns represent chromosomes. Types of CNVs are annotated by color, depending on if the deletion/duplication is detected in one sample (purple/blue) or two or more samples (green/yellow). (C) Kaplan-Meier plots showing the association between overall survival and alterations in at least $67 \%$ of the most frequent CNVs detected in LMS patients. (D) Heatmap of unsupervised hierarchical clustering based on the 370 genes affected with the most common CNVs related to patient outcome. (E) Proximal effects from the integrative analysis of CNVs and RNAseq data. Boxplots show a region's expression ( $y$-axis, $\log$ of normalized counts per million reads mapped) of genes regulated by the specific region ( $x$-axis) and colored by copy number state, represented as loss (blue), normal (orange), and gain (red) in LMS (upper) and LM (lower) samples. ** $p$-adjusted value $<0.01$; *** $p$-adjusted value $<0.001$. 


\subsection{Proximal Expression Effects Inferred from Integration with $\mathrm{CNV}$}

Because CNVs can involve a large region containing multiple genes, we integrated the detected CNV regions with exome-wide gene expression data from RNAseq in LM and LMS tumors (Supplementary Figure S3). As a result, we identified regions in the LMS samples located in chromosomes 5, 11,14, and 16 in which gains or losses were significantly associated with changes in expression involving five genes (Figure 2E). Specifically, in LMS (Figure 2E, upper), we detected TRIP13 with a positive correlation between gains and higher expression, while losses were associated with lower expression. However, the expression of other genes increased when there was a loss in the corresponding region $(C D K N 1 C)$ or decreased when there was a gain (BATF, DECR2, LUC7L), possibly due to different mechanisms of regulating gene expression. The same analysis was performed in the LM samples (Figure 2E, lower), showing regions located in chromosomes 5, 6, 7, 9, 11, 14, and 17. As in LMS, some genes showed a positive correlation between copy number state and expression (ZSCAN9, MARK3, CHRNB1, WRAP53, YBX2), while, in the remaining genes, gains in the chromosomal region were associated with lower expression values due to more complex gene regulation.

\subsection{Structural Rearrangements Affect Specific Regions and Genes in LM and LMS}

Further, we identified high-confidence fusion transcripts in $29.5 \%$ of the LM cases and $61.8 \%$ of LMS arising from chromosomal rearrangements, resembling chromothripsis in some cases, such as LMS25 and LMS26 (Figure 3A).

Chromosomes $3,8,11,13,17$, and $\mathrm{X}$ were the most frequently affected in LMS (Figure 3A), while chromosomes 1, 3, 6, and 14 were the most impacted in LM (Figure 3B). Although no recurrent fusions were detected in any tumors, multiple rearrangements targeting the chromatin remodeling protein ATRX were identified in LMS, and a known oncogene, RAD51B, was identified in LM. Specifically, ATRX was fused with several gene partners, such as TRAPPC9, RP11-56A10.1, and EZH1, resulting in a non-functional fusion protein lacking the helicase ATP-binding domain and/or the helicase C-terminal domain in the LMS tumors (Figure 3A). In the LM tumors, RAD51B was fused with HMGA2, NCOR2, and NUDT3, indicating the potential of these fusions to drive tumorigenesis (Figure $3 \mathrm{~B}$ ). 

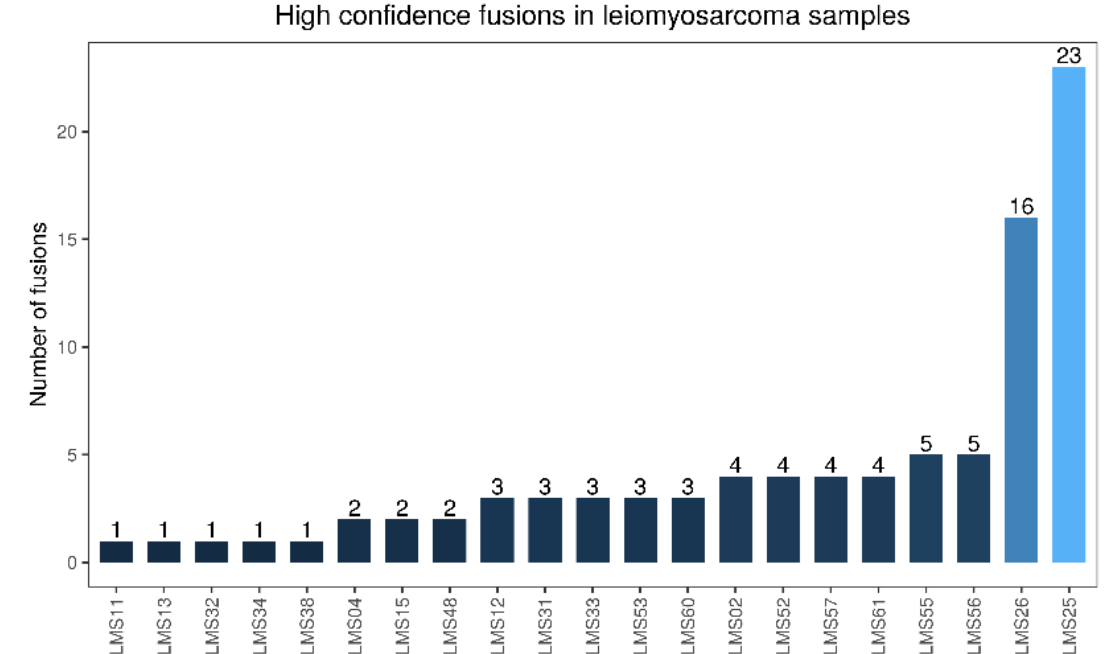

Frequently affected regions in LMS

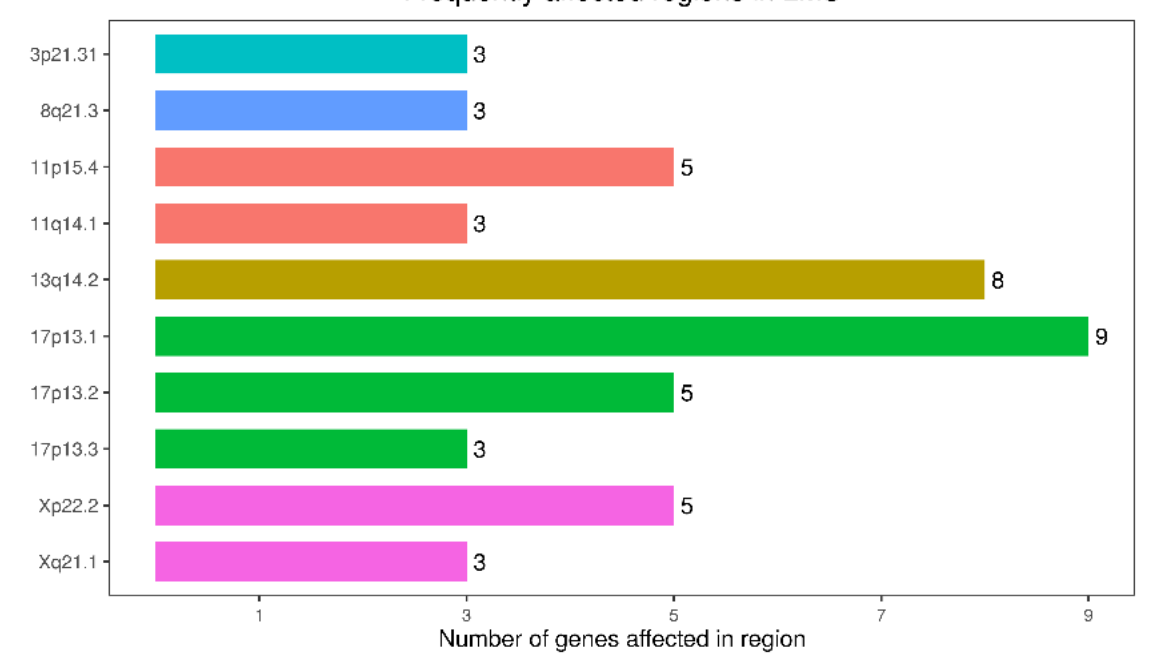

Figure 3. Cont
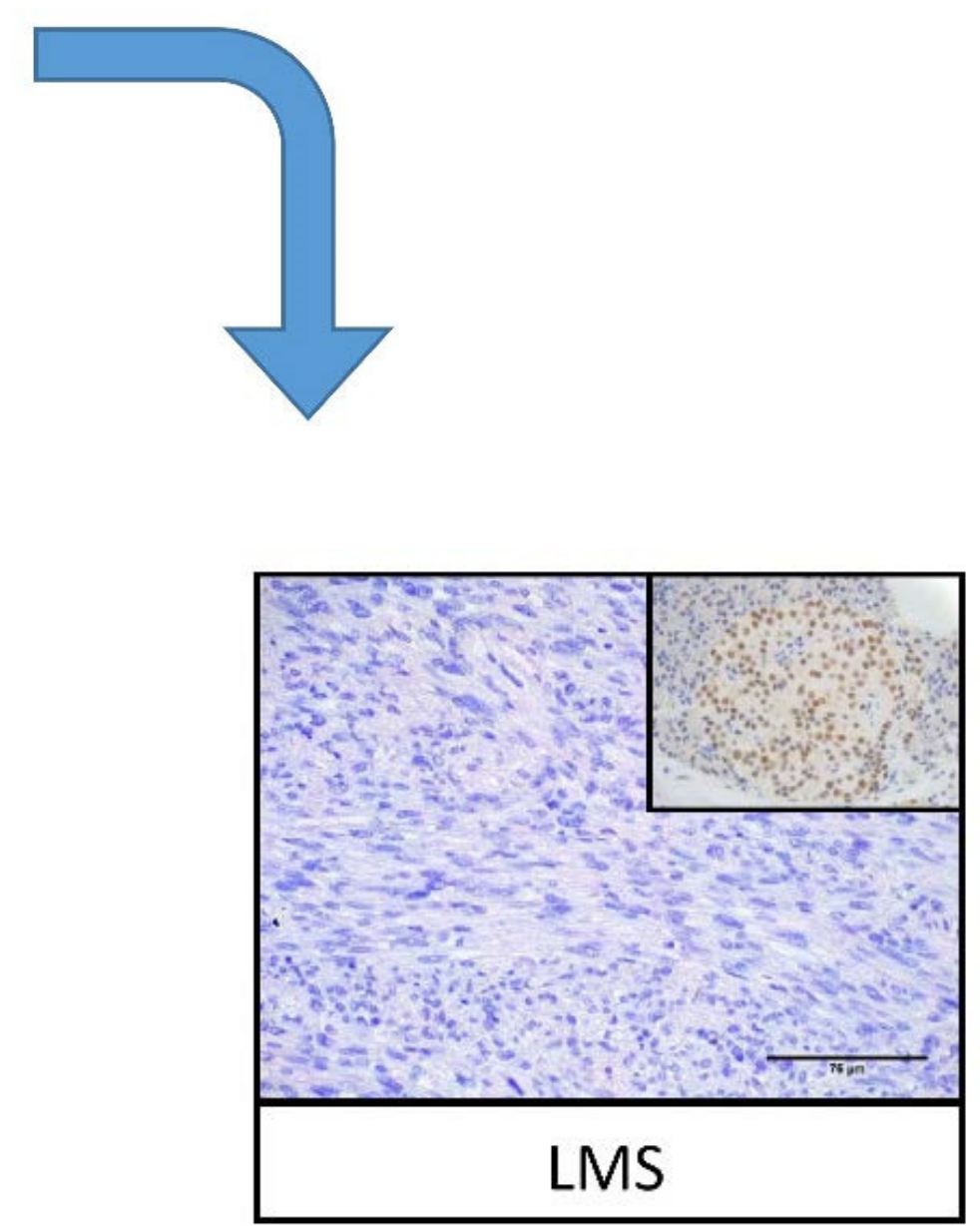

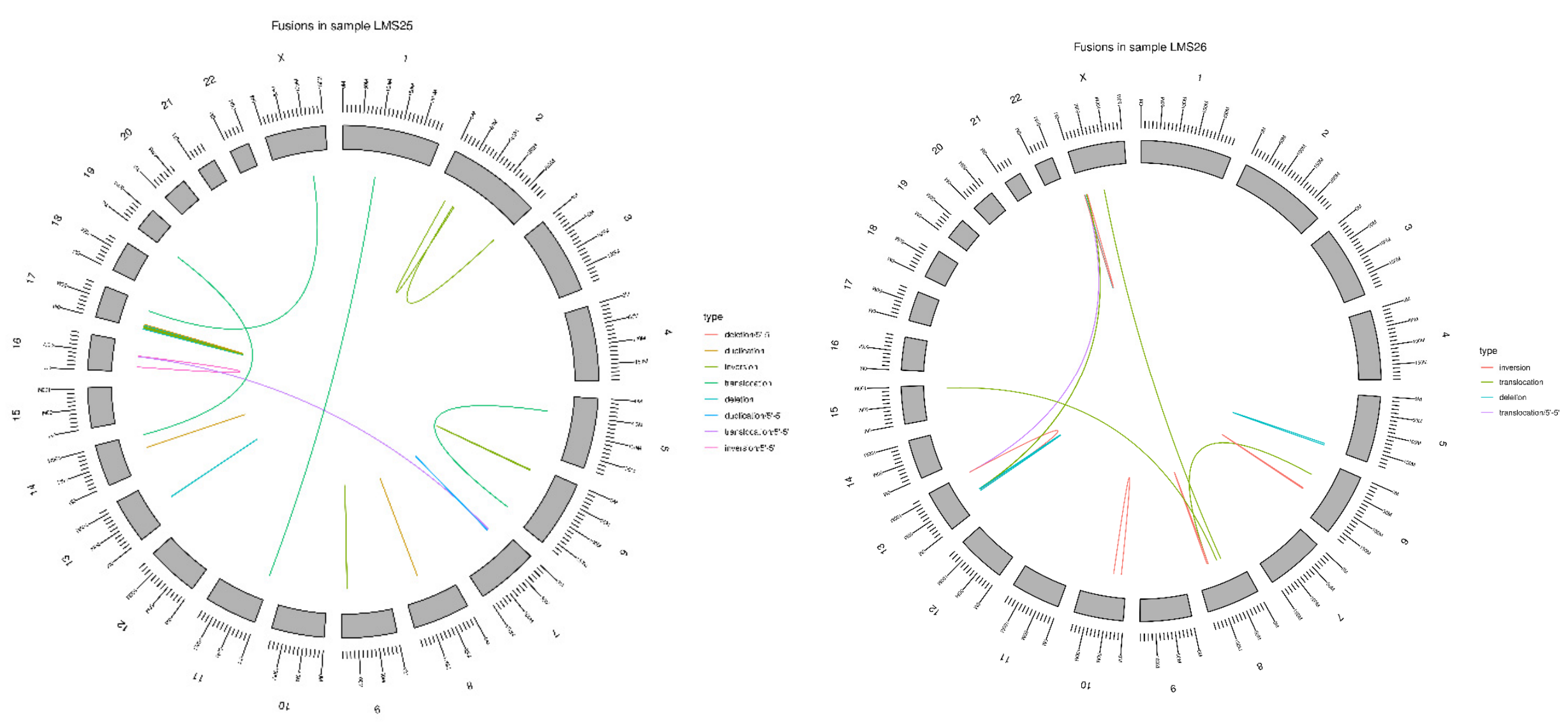

Figure 3. Cont. 


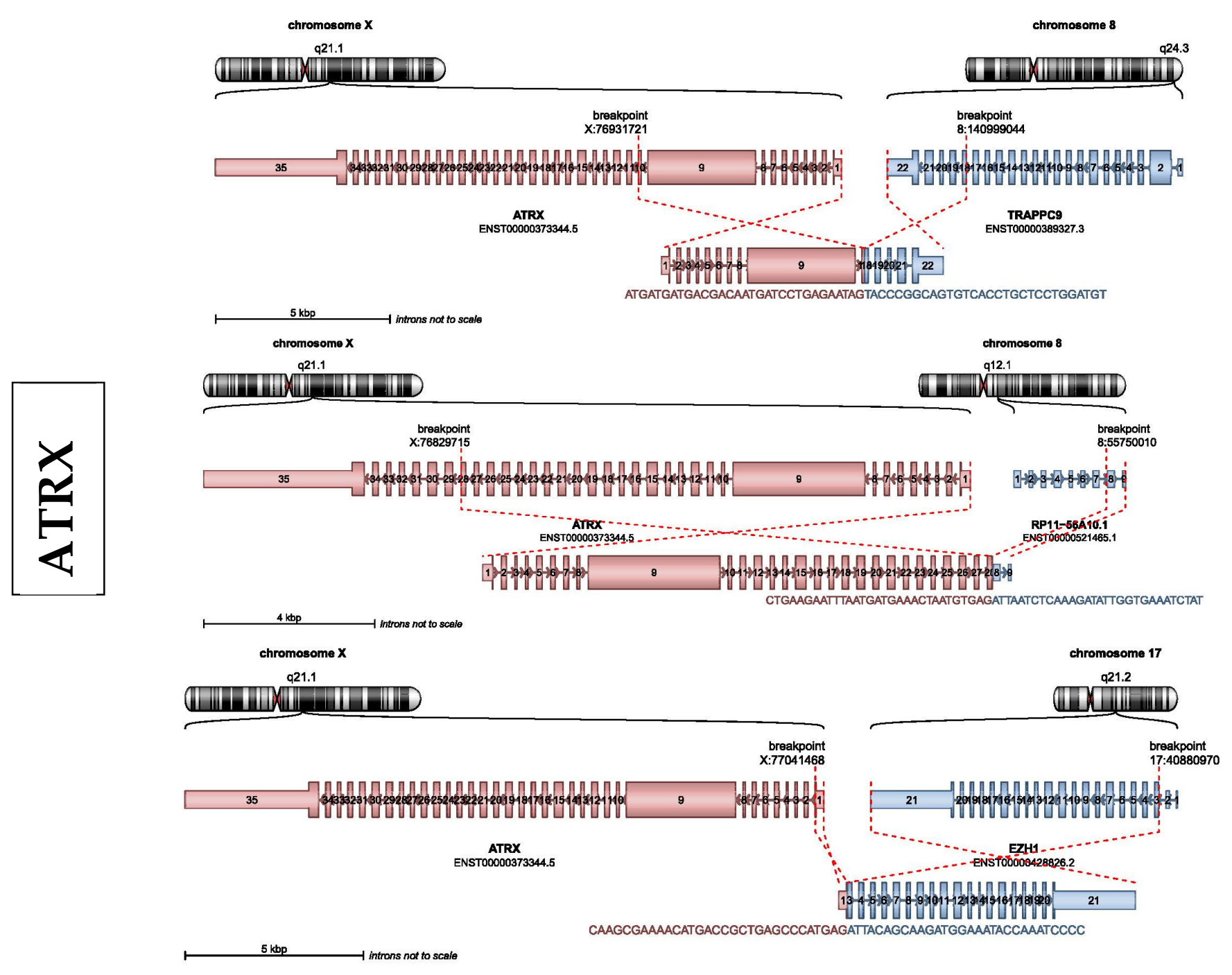

(A)

Figure 3. Cont. 


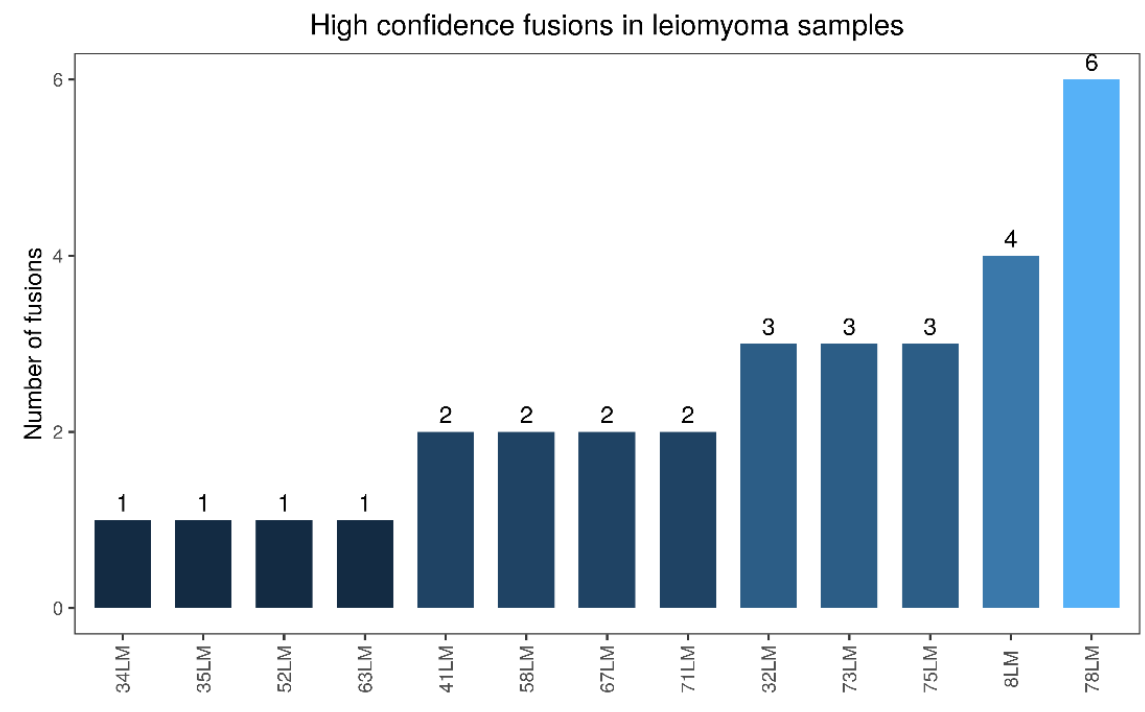

Frequently affected regions in LM
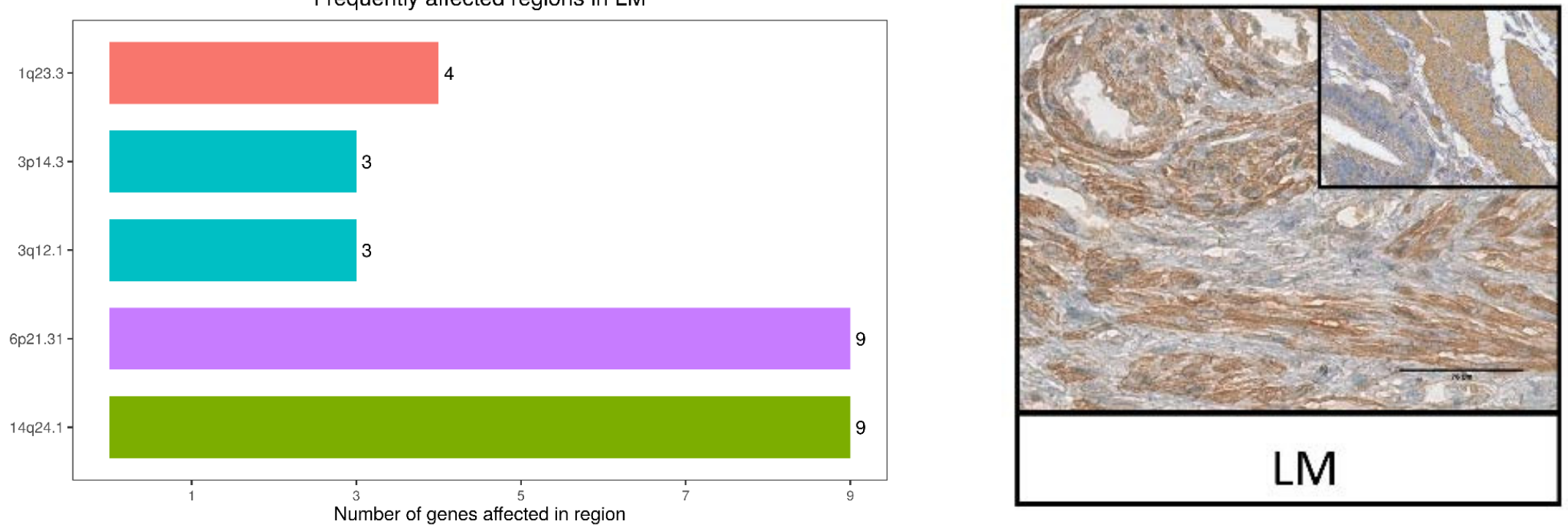

Figure 3. Cont. 


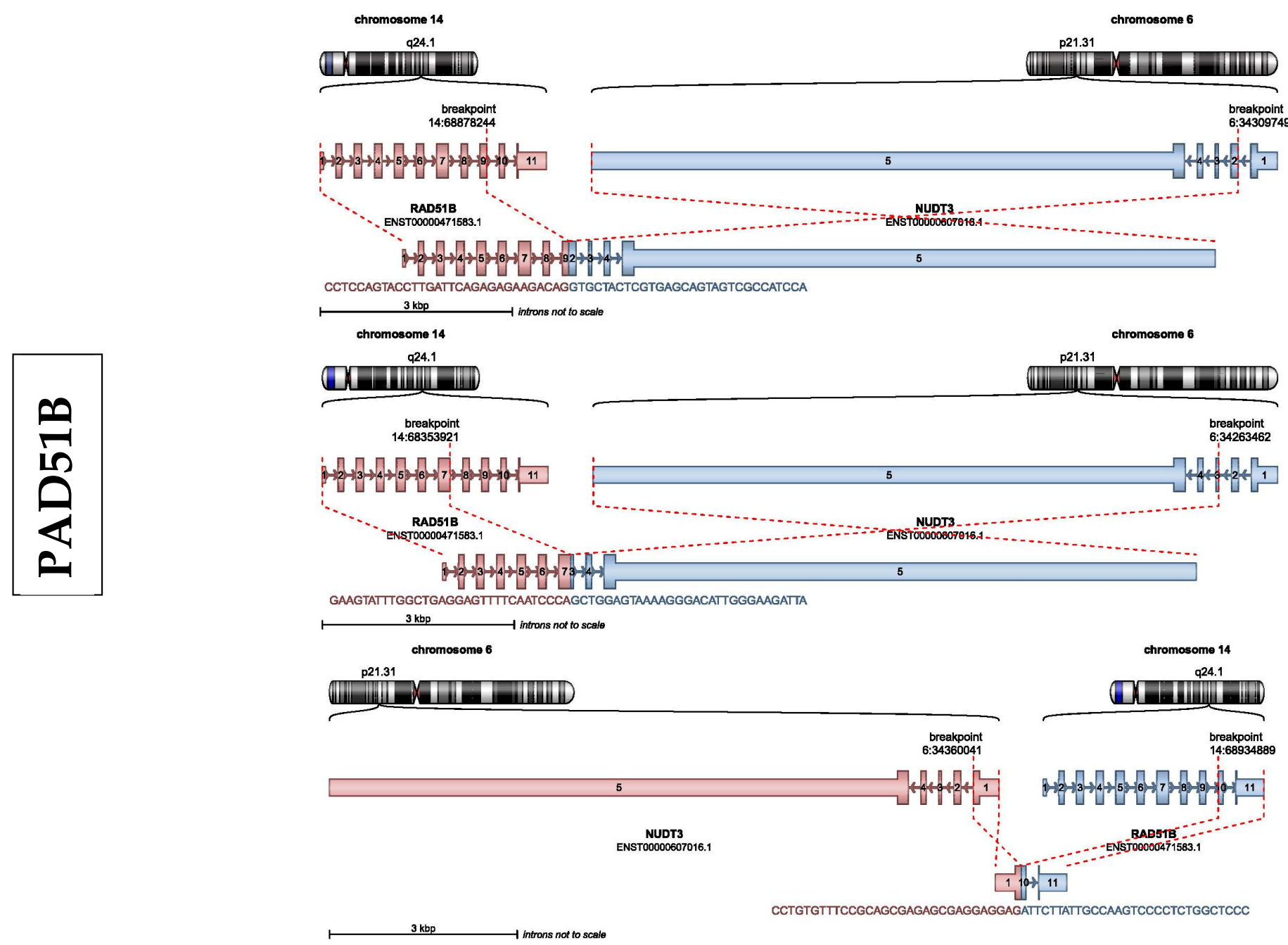

(B)

Figure 3. Structural variant plots of chromosomal rearrangements in leiomyoma (LM) and leiomyosarcoma (LMS). (A) Bar plot showing the number of high-confidence fusions per LMS sample (upper). Bar plot and ideograms showing the most frequently affected chromosome regions in LMS samples (middle). 
Schematic representation of the gene sequence and functional protein domain for the most affected gene, $A T R X$, validated by immunohistochemistry (lower), using glioma biopsies as a positive control (right). Scale bar represents $75 \mu \mathrm{M}(n=3)$. (B) Bar plot showing the number of high-confidence fusions per LM sample (upper). Bar plots and ideograms showing the most frequently affected chromosome regions in LM samples (middle). Schematic representation of the gene sequence and functional protein domain for the most affected gene, $R A D 51 B$, validated by immunohistochemistry (lower) and using gallbladder as a positive control (right). Scale bar represents $75 \mu \mathrm{M}(n=3)$. 


\subsection{Differential Transcriptomic Characterization of LMS versus LM}

We next sought to identify differential gene expression footprints by the RNAseq analysis of 44 LM and 34 LMS tumors. A class comparison detected a total of 489 DEGs, 416 significantly upregulated and 73 downregulated, between LMS and LM (FDR $<0.05$ and $|\log \mathrm{FC}|>2$ ). Some of the most significant upregulated genes in LMS were validated by RT-PCR, confirming significant overexpression and correlation between qPCR and RNAseq (Supplementary Figure S4).

Next, unsupervised hierarchical clustering grouped the LMS samples in a homogeneous cluster of 29 samples, while $30 \mathrm{LM}$ samples were detected in a separate cluster. Of note, another cluster included the remaining LM with some LMS (LMS03, LMS11, LMS26, LMS35, and LMS62) (Figure 4A). Based on the heatmap/dendrogram, these LMS samples appeared closer to the LM group, suggesting that their molecular profile was more similar to the LM samples than the LMS samples. This was in line with clinical information of the corresponding patients as only one out of the five LMS patients died due to the disease, while the other four are still alive, reinforcing that these tumors may have intermediate characteristics but are closer to LM.

To better understand the molecular functions, biological processes, and pathways differentially regulated by the 489 DEGs, we performed Kyoto Encyclopaedia of Genes and Genomes (KEGG), Gene Ontology (GO), and Reactome enrichment analyses. In total, we detected 10 KEGG pathways, 83 GO terms, and 92 Reactome pathways (Supplementary Figure S5). Briefly, the 489 DEGs were mostly involved in cell cycle-associated processes (e.g., nuclear division, chromosome segregation, regulation of cell cycle phase transition, meiosis) according to all the databases (Supplementary Figure S5). In summary, these results indicate that differences in the expression between LMS and LM occurred in genes associated mainly with cell cycle and nucleic acid metabolism, suggesting that alterations of these processes occur differently and have different consequences for each tumor type. 


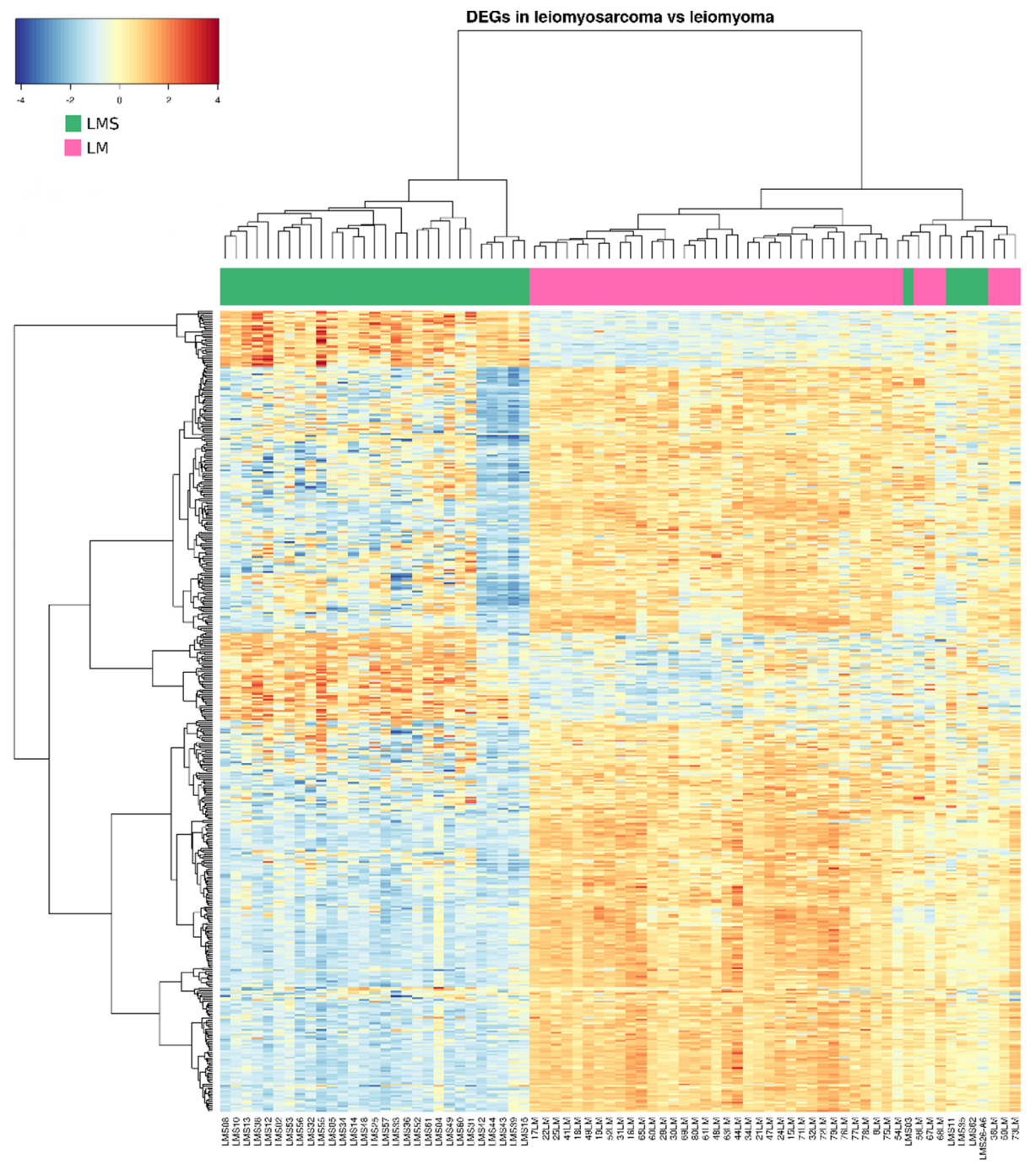

(A)

Figure 4. Cont. 


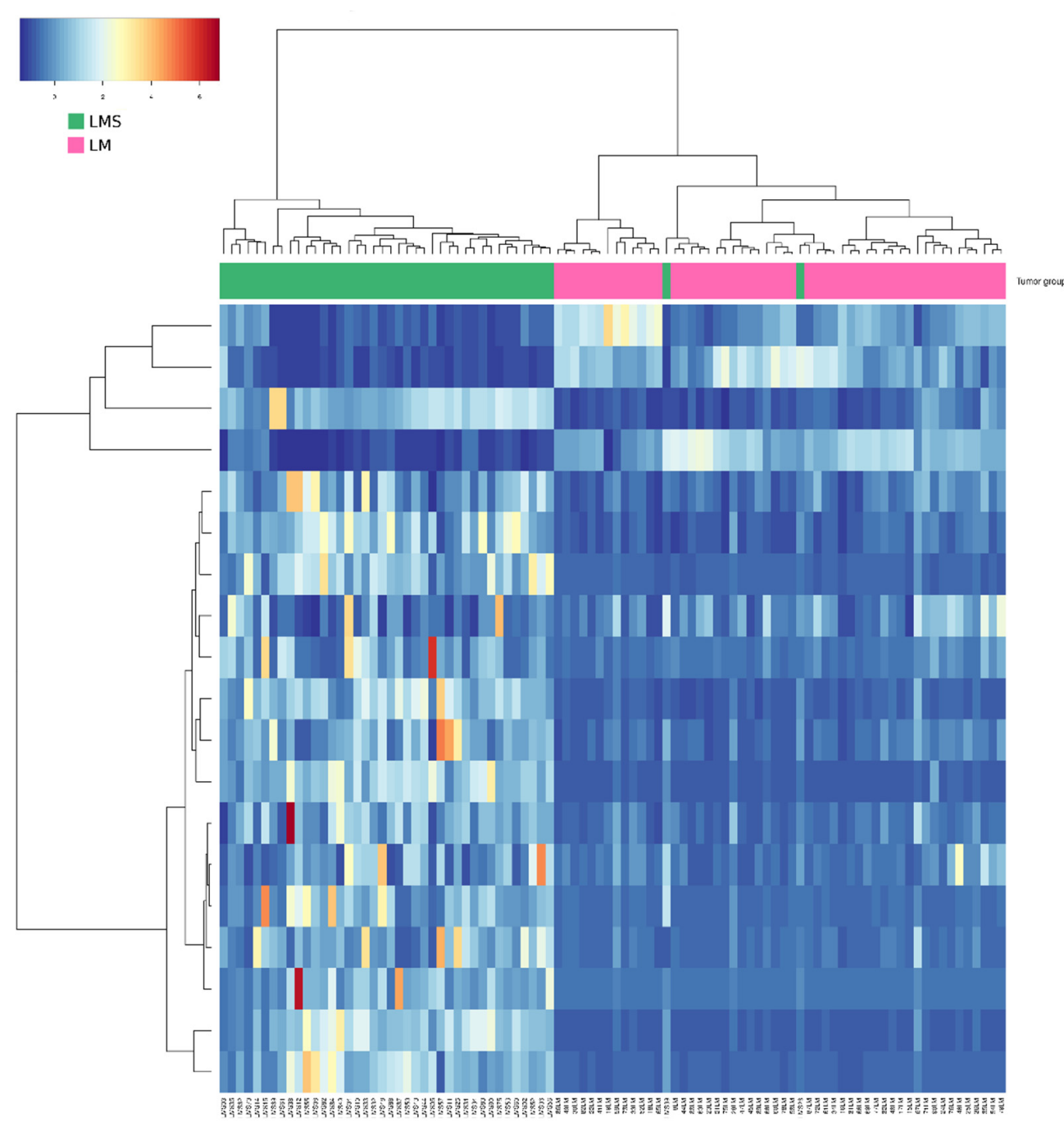

(B)

Figure 4. Cont 


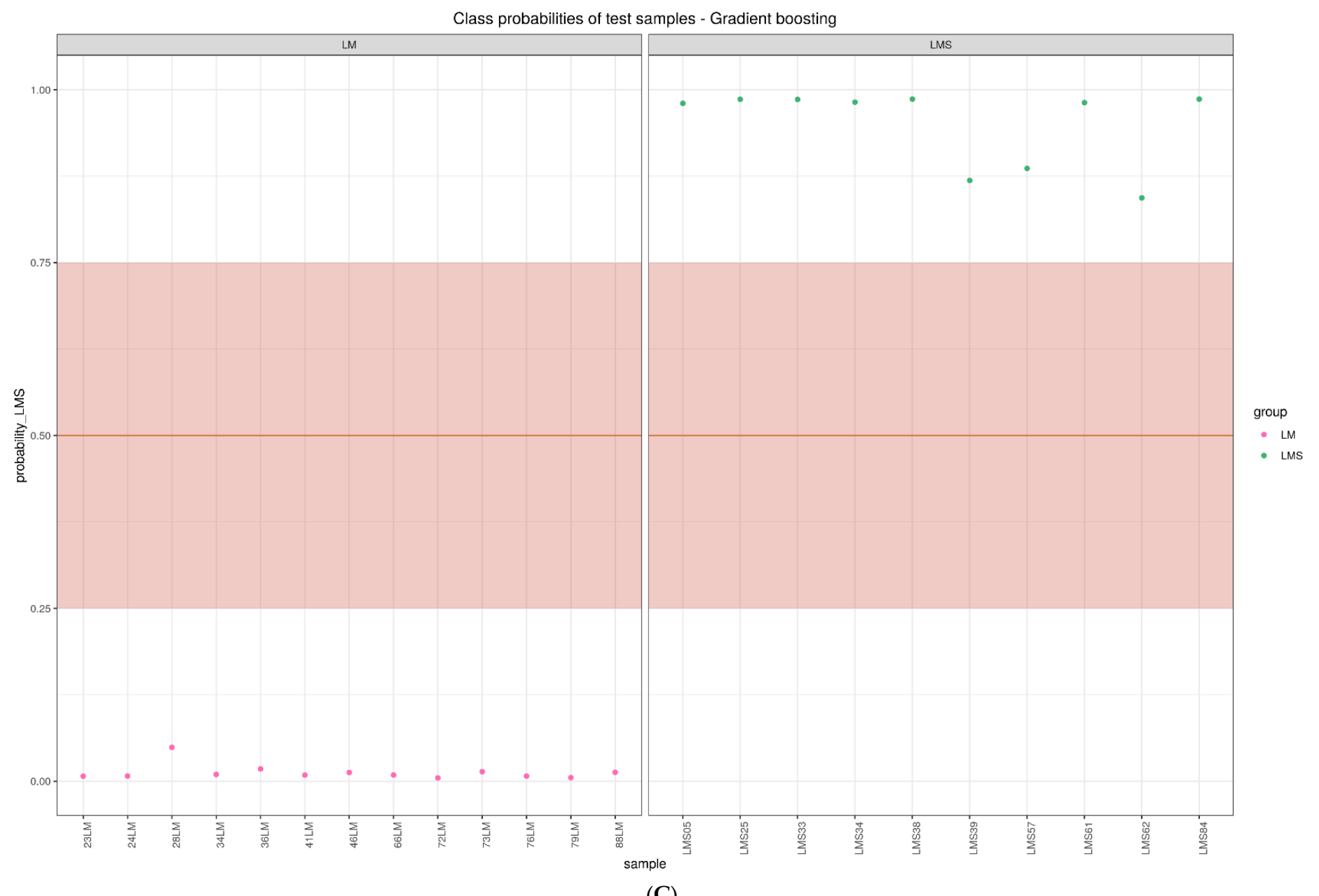

(C)

Figure 4. Transcriptional analysis and validation of the targeted gene panel on leiomyoma (LM) and leiomyosarcoma (LMS). (A) Heatmap of hierarchical clustering. Top dendrogram shows clustering of samples, and left dendrogram shows clustering of all differentially expressed genes. Colors in the heatmap represent gene expression intensities, with blue indicating low expression and red indicating high expression. The bar on top of the heatmap represents the group by color (green = LMS; pink = LM). (B) Heatmap showing clustering of samples using the normalized coverage data for each of the 19 genes. (C) Class probabilities predicted by the model for the test set, with the "warning range" highlighted in light orange. 


\subsection{Model Creation and Validation for Differential Molecular Diagnosis of LMS and LM}

To classify LM and LMS, we developed a machine learning approach based on the transcriptomic signatures of each group since we found that management, analysis, and biological comprehension were more straightforward when using RNAseq data. After feature pruning, the final model was composed of 19 DEGs and was able to correctly classify all the samples in the validation set.

Based on this model, we built a targeted sequencing panel using AmpliSeq technology for the 19 selected genes, which was used to re-analyze all the previous LM and LMS tumors ( $n=44$ and $n=32$, respectively, since two LMS samples were filtered due to poor sequencing quality) in addition to new samples (eight LM and ten LMS).

Next, the total 96 samples were randomly split into a training set to build the machine learning model and a test set to validate the model $(75 \%$ and $25 \%$ class-balanced samples for the training and test sets, respectively). Specifically, the gradient boosting algorithm was used to build a new model, which achieved optimal values of sensitivity and specificity since it was able to correctly classify all the test samples. The feature selection resulted in a final model that consisted only of 19 genes, out of which only three, COL4A5, MFAP5, and ITGA9, were overexpressed in leiomyoma, while the rest were overexpressed in leiomyosarcoma). The unsupervised clustering of the samples based on these 19 genes allowed separation through a heatmap of two distinguishable groups of LM and LMS. However, and resembling results from previous RNAseq analysis, two LMS (LMS26 and LMS39) were clustered in a group opposite to those confirmed by pathology (Figure 4B).

Further, the model was used to classify the samples and to calculate class probabilities, allowing a more fine-tuned classification of the samples, where we defined a "warning range" for those tumors where the model was not confident enough, defined as probabilities of $<75 \%$ for each group (Figure $4 \mathrm{C}$ ). Interestingly, this model could correctly classify all the samples with high class probabilities, even for sample LMS39 with the lowest LMS probability.

\section{Discussion}

The search for molecular criteria to differentiate uterine myometrial tumors represents an important current diagnostic challenge, where molecular profiling could be a powerful complement to current diagnosis based on the clinical presentation, imaging features, and microscopic morphologic characteristics. Since our main aim was to build a preliminary differential diagnostic tool, we have focused on the comparison between the most prevalent benign myometrial tumors, LM, and the most aggressive ones, LMS.

While we are aware that excluding other tumor types, such as STUMPs, inflammatory myofibroblastic tumors (IMT), or undifferentiated uterine sarcomas, is a relevant limitation of our study, we decided to discard these samples in order to avoid introducing noise in our molecular analysis, also considering that, given the low incidence of these tumors, the fact that excluding them would not have a significant impact on our preliminary classification tool.

Following a similar rationale, we chose to limit our analysis to only conventional LMS and LM given the low prevalence of non-conventional variants and their differences, which may also introduce noise in our analyses.

Consequently, in this study, exome- and transcriptome-wide analyses were performed on histologically confirmed conventional LM and LMS to investigate molecular differences between and within these two different entities.

In terms of small variants, we found that only a small percentage of variants $(27.63 \%)$ were shared by the two groups, while the rest were exclusive of LM or LMS, suggesting independent mechanisms for tumorigenesis. Interestingly, we found the c.2443C > T mutation in the REST gene involves the loss of the repressor in LM and promotes the aberrant expression of GPR10 in the PI3K/AKT mTOR pathway [15]. Conversely, in LMS, we detected three different mutations affecting the OBSCN gene, which has been proposed as a molecular tool to differentiate LMS from gastrointestinal stromal tumors within a two 
gene-classifier [16]. Likewise, mutations in CCDC68 involving microtubule organization have been reported in different cancer types $[17,18]$. Moreover, with variant information, we detected for the first-time signature 12 of COSMIC as a mutational mark in LM and LMS. While its etiology remains unknown, its presence has been associated with a small percentage $(<20 \%)$ of mutations in liver cancer [16].

Additionally, we found differences in the number and type of somatic CNVs, suggesting that they could be used for the differential diagnosis of myometrial tumors. Furthermore, we provided novel insights into the potential usefulness of CNVs as a prognostic biomarker for patients with LMS. However, the low number of samples available for the survival analysis (mainly due to a lack of data resulting from the use of commercial samples or censoring) is a strong limitation, and, thus, the tendency we observed should be carefully interpreted and further explored and validated.

Because CNVs may cause changes in transcription levels associated with cancer [17], we performed an integrative analysis on the $\mathrm{CNV}$ and expression data. Interestingly, we found that alterations in specific CNV regions in LMS affected the expression of CDKN1C, a cyclin/Cdk inhibitor that may act as a tumor suppressor [18].

Further, we screened transcriptomic data to detect fusions in LM and LMS. While no recurrent fusions were detected in LMS or LM, multiple rearrangements targeted ATRX and $R A D 51 B$, respectively. The loss of expression of $A T R X$ has been associated to the Alternative Lengthening of Telomeres phenotype, which allows tumor cells to escape programmed cell death. In uterine LMS, this mechanism has been associated with a poor prognosis and overall survival $[19,20]$. Additionally, we identified the RAD51B-HMGA2 fusion transcript in LM, which may have a role in tumor progression, as previously described [21].

The transcriptomic results revealed 489 differentially expressed in LMS versus LM. Although some of these genes have been reported $[11,22,23]$, we identified specific DEGs involved in mitotic spindle checkpoint regulation, including AURKA, SPAG5, NUF2, BUB1B, and KIF14 [24], while the CCDC68 gene affected by a deleterious variant mentioned in Section 2.1 is also involved in this process. The defective segregation of chromosomes and microtubule-kinetochore-spindle formation cause tumor cells to become aneuploid, allowing DNA-damaged cells to skip the spindle assembly checkpoint, suggesting a possible molecular mechanism for the development of LMS. One of the most significant genes was AURKA, a cell cycle protein that is also overexpressed in cervical [25] and ovarian [26] cancers and seems to be key in the pathogenesis of LMS since its inhibition results in cell cycle arrest and apoptosis in LMS cell lines [27].

Further analysis based on hierarchical clustering revealed some LMS samples that unexpectedly clustered with LM samples. Accordingly, after reviewing the clinical features and follow-up, we found that most of these patients exhibit higher overall survivals than reported by previous retrospective studies [28]. These findings are consistent with our prior premise that myometrial tumors can be differentiated based on their transcriptomic profiles.

We finally built a classification model composed of nineteen genes, sixteen overexpressed in LMS and three overexpressed in LM, possibly suggesting that the overexpression of a small subset of genes results in a more disrupted molecular profile that is sufficient to indicate that a tumor is more likely to be a malignant uterine leiomyosarcoma. The genes in this panel are involved in different processes and functions, including DNA replication (BRCA2, CHAF1A, E2F7, and EXO1); DNA damage repair (ARHGAP11A and PBK); extracellular matrix formation and interaction (COL4A5, ITGA9, and MFAP5), and, lastly, segregation of chromosomes and microtubule-kinetochore-spindle formation (CCDC34, CDCA5, CENPE, CENPF, CENPH, and MLF1IP), which, as we have mentioned, could be an affected pathway in the tumorigenesis of LMS. As a result, our model could be a representation of the affected pathways that differ between LMS and LM, where the genes associated with alterations in the extracellular matrix suggest the diagnosis of LM (since COL4A5, ITGA9, and MFAP5 are the only genes overexpressed in LM), and the genes associated with defective DNA replication or DNA damage repair or segregation of chromosomes suggest the diagnosis of LMS. 
Moreover, this model correctly classified all the samples in the validation set with high sensitivity and specificity, while the class probabilities calculated for each sample showed the predictions of the model were of high confidence (all $>75 \%$ for their class). It is also of note that, even though the unsupervised hierarchical clustering of global RNAseq showed a mixed cluster of LMS and LM samples, the machine learning algorithm effectively classified these samples, once again demonstrating the potential of the predictive tool.

Nevertheless, although promising, the genomic and transcriptomic outcomes are based on the analysis of the entire tumor, which implies a technical limitation due to intratumoral heterogeneity. Additional studies with larger numbers of samples are also needed to validate and clinically use this tumor classification model.

In summary, our findings provide a novel molecular profile and candidate gene targets to discriminate LM from LMS at the tumor-tissue level, establishing a potential diagnostic tool that could be helpful to complement morphological and immunohistochemical diagnostic features.

Accordingly, this molecular-driven test linked to functional histology might provide a framework for objective and specific diagnosis at the tumor tissue level. The challenge, however, for both the pathologist and the physician, is how best to effectively integrate this morphological and molecular information into a comprehensive diagnosis and treatment plan.

\section{Materials and Methods}

Detailed description of the materials and methods used in this study are provided in the Supplementary Materials and Methods (Appendix A).

\subsection{Clinical Sample Collection}

Use of human tissue samples was previously approved by the IRB of the hospitals involved: Hospital La Fe, Valencia, Spain (24 July 2019), Hospital Virgen de la Arrixaca Murcia, Spain (17 December 2019), Hospital Santa Lucía Murcia, Spain (28 January 2020), and Fundación Instituto Valenciano de Oncologia (IVO), Valencia, Spain (2 December 2020). All patients signed and provided written informed consent.

Briefly, formalin-fixed paraffin-embedded (FFPE) tumor samples were collected from 119 women undergoing hysterectomy or myomectomy as surgical treatment for primary myometrial tumors (Supplementary Figure S1). Before further processing, anonymized samples were evaluated by two pathologists with wide experience in gynecology, who histologically confirmed a diagnosis of LM or LMS according to WHO criteria [14] and provided us with paraffin blocks consisting of at least $85 \%$ of tumor tissue. Patients with other gynecological tumors, disorders, malignancies, or diagnosed bacterial, fungal, or viral infections were excluded $(n=16)$.

Specifically, Hospital la Fe contributed with 56 LM samples and 13 LMS tumors. Hospital Santa Lucía provided to this study 6 LMS, 12 LMS came through Hospital Virgen de la Arrixaca, while 3 LMS were provided by IVO, and 13 LMS were supplied by Origene Technologies Inc. (Rockville, MD, USA). Selected LM and LMS samples were then split into two cohorts: the experimental cohort, which was used to study global DNA and RNAseq profiles (44 LM, 34 LMS), and the validation cohort, including additional new samples (8 LM, 10 LMS) to perform targeted sequencing and model validation (Supplementary Figure S1). Epidemiological, histopathological, and clinical outcomes are summarized in Table 1. This study was registered on ClinicalTrials.gov (ID NCT04214457), and data were monitored by a clinical research associate.

\subsection{DNA Sequencing and Analysis}

After nucleic acid isolation, DNA libraries were constructed using the KAPA Hyper Prep kit (Roche, Basel, Switzerland) and enriched using a panel of 571 hematologicalassociated genes. DNA sequence data were demultiplexed and aligned to the human hg19 
genome (CRGCh37) using BWA [29] and SAMtools [30] following the quality control and metrics detailed in Supplementary Table S3.

Sequencing data were analyzed for small variants using Freebayes (https: / / github. $\mathrm{com} /$ freebayes/freebayes, accessed on 31 December 2021) and annotated with SnpEff (https:/ / github.com/pcingola/SnpEff, accessed on 31 December 2021). Additional assessment of somatic mutational signatures inferred from SNVs was done using MutationalPatterns [31]. Further analysis for microsatellite instability (MSI) was performed using a set of six mononucleotide repeat markers (Supplementary Table S4) following published protocols [32]. Lastly, we used CNVkit with default parameters (https:/ / github.com/etal/cnvkit, accessed on 31 December 2021), for CNV detection.

\subsection{RNA Sequencing and Analysis}

RNA libraries from 44 LM and 34 LMS were constructed using Truseq RNA exome (Illumina, San Diego, CA, USA) and aligned to the human hg19 genome using STAR (https:/ / github.com/alexdobin/STAR, accessed on 31 December 2021) (Supplementary Table S5) to estimate gene transcript abundance with HTseq (https:/ / github.com/simonanders/htseq, accessed on 31 December 2021).

First, arriba (https://github.com/suhrig/arriba/, accessed on 31 December 2021) was used for detection of fusions on RNAseq data, which were then validated by immunohistochemistry. Subsequently, differential expression analysis between LMS and LM was performed using edgeR [33] and subjected to functional analysis. Some of the most significant DEGs were finally validated using RT-qPCR (Supplementary Table S6).

\subsection{Integrative DNA/RNA Analysis}

To evaluate the association between CNVs and RNAseq counts, we used CNVRanger (https:/ / github.com/waldronlab/CNVRanger, accessed on 31 December 2021), excluding genes with $<20$ counts per million (cpm) and $\mathrm{CNV}$ regions with $<10$ samples in a group deviating from $2 \mathrm{n}$, with a $1 \mathrm{Mbp}$ window and $p$-value $<0.01$ (Supplementary Figure S3).

\subsection{Building and Validating the Classification Model}

Since we found that analysis and clinical interpretation were more straightforward when using RNAseq, we built a classification model with this data using caret package (https://github.com/topepo/ caret, accessed on 31 December 2021). For this purpose, our sample cohort was randomly split, keeping balanced class distributions into a training (75\% of samples) and test set (25\% of samples). We used the Adaboost algorithm on DEGs cpm to perform a prior selection of predictor genes.

Validation of the model was next performed by re-sequencing LM $(n=44)$ and LMS $(n=34)$ samples, adding a new set of 8 LM and 10 LMS samples. Briefly, RNA from 96 FFPE tissue sections was used to prepare libraries with a PCR/amplicon-based workflow (AmpliSeq Library Plus, Illumina, San Diego, CA, USA).

Normalized coverage values were introduced in caret using the gradient boosting algorithm [34]. Once the model was built, the test set was used to construct receiver operating characteristic curves (ROCs), also assessing sensitivity and specificity (Supplementary Figure S6).

\subsection{Statistical Analyses}

Statistical analyses were performed using R (http:/ / www.R-project.org, accessed on 31 December 2021). Two-tailed Student's $t$-tests were used to compare quantitative clinical variables in LMS and LM patients and for gene validation using RT-qPCR. All survival analyses were achieved by Cox regression in a multivariate model to account for LMS prognostic factors and compared between groups using the log-rank test. Correlation between RNAseq and qPCR-based $\log F C$ was evaluated using Pearson's correlation test. 


\section{Conclusions}

In conclusion, this study provides a novel molecular classification for leiomyoma and leiomyosarcoma tumors and may be a helpful tool for current diagnosis, hopefully laying the foundation for the future evaluation of malignancy risk.

\section{Patents}

A patent disclosure has been filed for the study under the inventors A.M., R.A., and C.S. since 11 October 2021.

Supplementary Materials: The following supporting information can be downloaded at: https: //www.mdpi.com/article/10.3390/ijms23042190/s1.

Author Contributions: Conceptualization: A.M. and C.S. Formal analysis: A.M.-L., R.A. and J.J.-A. Data curation: A.M. and M.G. Investigation: A.M.-L., A.M., S.L., A.T. and F.B. Resources: V.L., J.M., S.L., A.T., S.O., M.G., F.B. and S.D. Validation: S.L., A.T. and F.B. Writing-Original Draft: A.M. and A.M.-L. Writing - Review \& Editing: A.M. and C.S. Supervision: A.M. and C.S. Funding acquisition: A.M. All the authors have substantively revised the manuscript and approved the submitted version. All authors have read and agreed to the published version of the manuscript.

Funding: This research was funded by Miguel Servet Spanish Program Grant, grant number CP19/00162 and Health Research Funds, grant number PI20/00942 from Carlos III Institute, Spain (AM), as well as Generalitat Valenciana, grant number FDEGENT/2019/010 PhD Training Grant for Valencian Entities (AML/AM).

Institutional Review Board Statement: The study was conducted in accordance with the Declaration of Helsinki, registered on ClinicalTrials.gov (ID NCT04214457), and approved by the Institutional Review Board of Hospital La Fe, Valencia, Spain (24 July 2019), Hospital Virgen de la Arrixaca Murcia, Spain (17 December 2019), Hospital Santa Lucía Murcia, Spain (28 January 2020), and Fundación Instituto Valenciano de Oncologia (IVO), Valencia, Spain (2 December 2020).

Informed Consent Statement: Informed consent was obtained from all subjects involved in the study.

Data Availability Statement: The data presented in this study are available on request from the corresponding author due to ethical/privacy restrictions.

Acknowledgments: The authors thank the Biobank of Fundación Instituto Valenciano de Oncologia (IVO), Valencia, Spain for the human specimens used in this study. The authors also thank all members of the Gynecology and Pathology departments from Hospital La Fe (Valencia), especially Drs. Marina Tiermes, Violeta Moliner, and Beatriz Montero, as well as Aníbal Nieto from Hospital Virgen de la Arrixaca (Murcia). The authors acknowledge the technical support of Diana Valbuena and Carlos Gómez from the medical department of Igenomix R\&D, as well as Marta Gálvez, Tamara Garrido, and Patricia Escorcia for their experimental support.

Conflicts of Interest: C.S. is Founder and Head of the Scientific Advisory Board of Igenomix. J.J.-A., M.G., as well as R.A. are also employed by Igenomix SL. The remaining authors declare no competing interests.

\section{Abbreviations}

$\begin{array}{ll}\text { CNV } & \text { Copy Number Variant } \\ \text { COSMIC } & \text { Catalogue of Somatic Mutations in Cancer } \\ \text { CPM } & \text { Counts Per Million } \\ \text { DEGs } & \text { Differentially Expressed Genes } \\ \text { DNA } & \text { Deoxyribonucleic acid } \\ \text { FDA } & \text { Food and Drug Administration } \\ \text { FFPE } & \text { Formalin-fixed Paraffin-embedded } \\ \text { GO } & \text { Gene Ontology } \\ \text { Indel } & \text { Insertions/deletions } \\ \text { IMT } & \text { Inflammatory Myofibroblastic Tumor } \\ \text { IRB } & \text { Institutional Review Board }\end{array}$




$\begin{array}{ll}\text { KEGG } & \text { Kyoto Encyclopedia of Genes and Genomes } \\ \text { LM } & \text { Leiomyoma } \\ \text { LMS } & \text { Leiomyosarcoma } \\ \text { LogFC } & \text { Logarithm of Fold Change } \\ \text { MSI } & \text { Microsatellite instability } \\ \text { RNA } & \text { Ribonucleic acid } \\ \text { ROC } & \text { Receiver Operating Characteristic Curve } \\ \text { RT-qPCR } & \text { Real-time quantitative polymerase chain reaction } \\ \text { SNV } & \text { Single nucleotide variant } \\ \text { STUMP } & \text { Smooth uterine muscle of uncertain malignant potential } \\ \text { WHO } & \text { World Health Organization }\end{array}$

\section{Appendix A}

Appendix A contains an in-detail description of the materials and methods used in this study, including nucleic acid isolation, quality control, and quantification; DNA library preparation, targeted exome sequencing, and mapping; RNA library preparation, sequencing, and mapping; detection of chromosomal rearrangements and validation; differential expression analysis, validation by qRT-PCR and functional analysis, and gene selection for classification model and validation.

\section{References}

1. Baird, D.; Dunson, D.; Hill, M.; Cousins, D.; Schectman, J. High cumulative incidence of uterine leiomyoma in black and white women: Ultrasound evidence. Am. J. Obstet. Gynecol. 2003, 188, 100-107. [CrossRef] [PubMed]

2. $\quad$ Parker, W.; Berek, J.S.; Pritts, E.; Olive, D.; Kaunitz, A.M.; Chalas, E.; Clarke-Pearson, D.; Goff, B.; Bristow, R.; Taylor, H.S.; et al. An Open Letter to the Food and Drug Administration Regarding the Use of Morcellation Procedures in Women Having Surgery for Presumed Uterine Myomas. J. Minim. Invasive Gynecol. 2016, 23, 303-308. [CrossRef] [PubMed]

3. Halaska, M.J.; Haidopoulos, D.; Guyon, F.; Morice, P.; Zapardiel, I.; Kesic, V. European Society of Gynecological Oncology Statement on Fibroid and Uterine Morcellation. Int. J. Gynecol. Cancer 2017, 27, 189-192. [CrossRef]

4. Mas, A.; Simon, C. Molecular differential diagnosis of uterine leiomyomas and leiomyosarcomas. Biol. Reprod. 2019, 101, 1115-1123. [CrossRef]

5. Bhave Chittawar, P.; Franik, S.; Pouwer, A.W.; Farquhar, C. Minimally invasive surgical techniques versus open myomectomy for uterine fibroids. Cochrane Database Syst. Rev. 2014, 10, Cd004638. [CrossRef]

6. $\quad$ Siedhoff, M.T.; Doll, K.M.; Clarke-Pearson, D.L.; Rutstein, S.E. Laparoscopic hysterectomy with morcellation vs abdominal hysterectomy for presumed fibroids: An updated dec.cision analysis following the 2014 Food and Drug Administration safety communications. Am. J. Obstet. Gynecol. 2017, 216, 259.e251-259.e256. [CrossRef]

7. Seagle, B.L.; Alexander, A.L.; Strohl, A.E.; Shahabi, S. Discussing sarcoma risks during informed consent for nonhysterectomy management of fibroids: An unmet need. Am. J. Obstet. Gynecol. 2018, 218, 103.e1-103.e5. [CrossRef]

8. Ricci, S.; Stone, R.L.; Fader, A.N. Uterine leiomyosarcoma: Epidemiology, contemporary treatment strategies and the impact of uterine morcellation. Gynecol. Oncol. 2017, 145, 208-216. [CrossRef]

9. Hodgson, B. AAGL practice report: Morcellation during uterine tissue extraction. J. Minim. Invasive Gynecol. 2014, 21, 517-530. [CrossRef]

10. Amant, F.; Van den Bosch, T.; Vergote, I.; Timmerman, D. Morcellation of uterine leiomyomas: A plea for patient triage. Lancet Oncol. 2015, 16, 1454-1456. [CrossRef]

11. Quade, B.J.; Wang, T.Y.; Sornberger, K.; Dal Cin, P.; Mutter, G.L.; Morton, C.C. Molecular pathogenesis of uterine smooth muscle tumors from transcriptional profiling. Genes Chromosomes Cancer 2004, 40, 97-108. [CrossRef]

12. Makinen, N.; Kampjarvi, K.; Frizzell, N.; Butzow, R.; Vahteristo, P. Characterization of MED12, HMGA2, and FH alterations reveals molecular variability in uterine smooth muscle tumors. Mol. Cancer 2017, 16, 101. [CrossRef]

13. Garcia, N.; Al-Hendy, A.; Baracat, E.; Carvalho, K.; Yang, Q. Targeting Hedgehog Pathway and DNA Methyltransferases in Uterine Leiomyosarcoma Cells. Cells 2020, 10, 53. [CrossRef]

14. WHO Classification of Tumours Editorial Board. Female Genital Tumours, 5th ed.; Herrington, C., Ed.; International Agency for Research on Cancer: Lyon, France, 2020; Volume 4.

15. Forbes, S.; Beare, D.; Boutselakis, H.; Bamford, S.; Bindal, N.; Tate, J.; Cole, C.; Ward, S.; Dawson, E.; Ponting, L.; et al. COSMIC: Somatic cancer genetics at high-resolution. Nucleic Acids Res. 2017, 45, D777-D783. [CrossRef] [PubMed]

16. Letouzé, E.; Shinde, J.; Renault, V.; Couchy, G.; Blanc, J.; Tubacher, E.; Bayard, Q.; Bacq, D.; Meyer, V.; Semhoun, J.; et al. Mutational signatures reveal the dynamic interplay of risk factors and cellular processes during liver tumorigenesis. Nat. Commun. 2017, 8, 1315. [CrossRef] [PubMed]

17. Almal, S.; Padh, H. Implications of gene copy-number variation in health and diseases. J. Hum. Genet. 2012, 57, 6-13. [CrossRef] [PubMed] 
18. Dobashi, Y.; Noguchi, T.; Nasuno, S.; Katayama, K.; Kameya, T. CDK-inhibitors-associated kinase activity: A possible determinant of malignant potential in smooth muscle tumors of the external soft tissue. Int. J. Cancer 2001, 94, 353-362. [CrossRef]

19. Liau, J.Y.; Tsai, J.H.; Jeng, Y.M.; Lee, J.C.; Hsu, H.H.; Yang, C.Y. Leiomyosarcoma with alternative lengthening of telomeres is associated with aggressive histologic features, loss of ATRX expression, and poor clinical outcome. Am. J. Surg. Pathol. 2015, 39, 236-244. [CrossRef]

20. Ahvenainen, T.; Mäkinen, N.; von Nandelstadh, P.; Vahteristo, M.; Pasanen, A.; Bützow, R.; Vahteristo, P. Loss of ATRX/DAXX expression and alternative lengthening of telomeres in uterine leiomyomas. Cancer 2018, 124, 4650-4656. [CrossRef]

21. Mehine, M.; Kaasinen, E.; Heinonen, H.R.; Mäkinen, N.; Kämpjärvi, K.; Sarvilinna, N.; Aavikko, M.; Vähärautio, A.; Pasanen, A.; Bützow, R.; et al. Integrated data analysis reveals uterine leiomyoma subtypes with distinct driver pathways and biomarkers. Proc. Natl. Acad. Sci. USA 2016, 113, 1315-1320. [CrossRef]

22. Adams, C.L.; Dimitrova, I.; Post, M.D.; Gibson, L.; Spillman, M.A.; Behbakht, K.; Bradford, A.P. Identification of a novel diagnostic gene expression signature to discriminate uterine leiomyoma from leiomyosarcoma. Exp. Mol. Pathol. 2019, 110, 104284. [CrossRef] [PubMed]

23. Kun, E.; Tsang, Y.; Lin, S.; Pan, S.; Medapalli, T.; Malpica, A.; Richards, J.; Gershenson, D.; Wong, K. Differences in gynecologic tumor development in Amhr2-Cre mice with KRAS G12D or KRAS G12V mutations. Sci. Rep. 2020, 10, 20678. [CrossRef] [PubMed]

24. Tien, A.C.; Lin, M.H.; Su, L.J.; Hong, Y.R.; Cheng, T.S.; Lee, Y.C.; Lin, W.J.; Still, I.H.; Huang, C.Y. Identification of the substrates and interaction proteins of aurora kinases from a protein-protein interaction model. Mol. Cell. Proteom. 2004, 3, 93-104. [CrossRef] [PubMed]

25. Twu, N.F.; Yuan, C.C.; Yen, M.S.; Lai, C.R.; Chao, K.C.; Wang, P.H.; Wu, H.H.; Chen, Y.J. Expression of Aurora kinase A and B in normal and malignant cervical tissue: High Aurora A kinase expression in squamous cervical cancer. Eur. J. Obstet. Gynecol. Reprod. Biol. 2009, 142, 57-63. [CrossRef]

26. Gritsko, T.M.; Coppola, D.; Paciga, J.E.; Yang, L.; Sun, M.; Shelley, S.A.; Fiorica, J.V.; Nicosia, S.V.; Cheng, J.Q. Activation and overexpression of centrosome kinase BTAK/Aurora-A in human ovarian cancer. Clin. Cancer Res. 2003, 9, $1420-1426$.

27. Shan, W.; Akinfenwa, P.Y.; Savannah, K.B.; Kolomeyevskaya, N.; Laucirica, R.; Thomas, D.G.; Odunsi, K.; Creighton, C.J.; Lev, D.C.; Anderson, M.L. A small-molecule inhibitor targeting the mitotic spindle checkpoint impairs the growth of uterine leiomyosarcoma. Clin. Cancer Res. 2012, 18, 3352-3365. [CrossRef]

28. Seagle, B.L.; Sobecki-Rausch, J.; Strohl, A.E.; Shilpi, A.; Grace, A.; Shahabi, S. Prognosis and treatment of uterine leiomyosarcoma: A National Cancer Database study. Gynecol. Oncol. 2017, 145, 61-70. [CrossRef]

29. Li, H.; Durbin, R. Fast and accurate short read alignment with Burrows-Wheeler transform. Bioinformatics 2009, 25, 1754-1760. [CrossRef]

30. Li, H.; Handsaker, B.; Wysoker, A.; Fennell, T.; Ruan, J.; Homer, N.; Marth, G.; Abecasis, G.; Durbin, R.; Genome Project Data Processing, S. The Sequence Alignment/Map format and SAMtools. Bioinformatics 2009, 25, 2078-2079. [CrossRef]

31. Alexandrov, L.B.; Nik-Zainal, S.; Wedge, D.C.; Aparicio, S.A.; Behjati, S.; Biankin, A.V.; Bignell, G.R.; Bolli, N.; Borg, A.; Borresen-Dale, A.L.; et al. Signatures of mutational processes in human cancer. Nature 2013, 500, 415-421. [CrossRef]

32. Pagin, A.; Zerimech, F.; Leclerc, J.; Wacrenier, A.; Lejeune, S.; Descarpentries, C.; Escande, F.; Porchet, N.; Buisine, M.P. Evaluation of a new panel of six mononucleotide repeat markers for the detection of DNA mismatch repair-deficient tumours. Br. J. Cancer 2013, 108, 2079-2087. [CrossRef] [PubMed]

33. Robinson, M.D.; McCarthy, D.J.; Smyth, G.K. edgeR: A Bioconductor package for differential expression analysis of digital gene expression data. Bioinformatics 2010, 26, 139-140. [CrossRef] [PubMed]

34. Friedman, J.H. Greedy function approximation: A gradient boosting machine. Ann. Stat. 2001, 29, 1189-1232. [CrossRef] 